\title{
QCD analysis of polarized deep inelastic scattering data
}

\author{
Johannes Blümlein*, Helmut Böttcher \\ Deutsches Elektronen Synchrotron, DESY, Platanenallee 6, D-15738 Zeuthen, Germany
}

Received 17 May 2010; received in revised form 26 July 2010; accepted 6 August 2010

Available online 11 August 2010

\begin{abstract}
A QCD analysis of the world data on polarized deep inelastic scattering is presented in next-to-leading order, including the heavy flavor Wilson coefficient in leading order in the fixed flavor number scheme. New parameterizations are derived for the quark and gluon distributions and the value of $\alpha_{S}\left(M_{z}^{2}\right)$ is determined. The impact of the variation of both the renormalization and factorization scales on the distributions and the value of $\alpha_{s}$ is studied. We obtain $\alpha_{s}^{\mathrm{NLO}}\left(M_{Z}^{2}\right)=0.1132_{-0.0095}^{+0.0056}$. The first moments of the polarized twist-2 parton distribution functions are calculated with correlated errors to allow for comparisons with results from lattice QCD simulations. Potential higher twist contributions to the structure function $g_{1}\left(x, Q^{2}\right)$ are determined both for proton and deuteron targets.
\end{abstract}

(c) 2010 Elsevier B.V. All rights reserved.

\section{Introduction}

The short-distance structure of the nucleon spin still consists a developing field. Nucleons as composite fermions obtain their spin in terms of a superposition of the spins and orbital angular momenta of their constituents, the quarks and gluons. It came as a surprise when the European Muon Collaboration (EMC) published its result [1] more than 20 years ago, which showed that the quarks do contribute only by a small fraction to the nucleon's spin. The obvious conclusion was to assume that the spin of the gluons and the orbital angular momenta of all constituents have to account for the missing fraction. This result initiated activities worldwide both on the experimental and the theoretical side in order to understand this spin puzzle and, finally, the spin structure of the nucleon.

\footnotetext{
* Corresponding author.

E-mail address: johannes.bluemlein@desy.de (J. Blümlein).
} 
Experiments performed at CERN, SLAC, DESY and JLAB [1-15] have contributed a vast amount of experimental data on inclusive polarized deeply inelastic lepton-nucleon scattering (DIS) during the last years. The main interest in measuring the short distance structure of polarized nucleons has somewhat moved from determining the first moments of the twist- 2 parton distributions to the extraction of their $x$-dependence in the measured region and their scaling violations due to QCD-evolution. At large enough four-momentum transfer $Q^{2}=-q^{2}$, the structure function $g_{1}\left(x, Q^{2}\right)$ mainly receives twist- 2 contributions ${ }^{1}$ and is related to the polarized twist-2 parton distributions.

In the present paper a QCD analysis of the polarized deep-inelastic world data is performed at next-to-leading order (NLO). Due to a larger set of new data which has become available recently the present analysis extends and updates earlier investigations [9,15,17-27]. In the QCDfit we determine the flavor singlet and non-singlet contributions of the polarized parton densities together with the QCD-scale $\Lambda_{\mathrm{QCD}}$ with correlated errors. The measurement of the strong coupling constant $\alpha_{S}\left(M_{Z}^{2}\right)$ from polarized deep-inelastic data does not reach the same precision as in the unpolarized case [28-31] since the measurement is based on an asymmetry and the present analysis is performed in NLO. However, a consistent analysis requires the determination of the QCD-scale $\Lambda_{\mathrm{QCD}}$ along with the parameters of the non-perturbative input distributions. Also, it is interesting to see which value of $\alpha_{s}\left(M_{Z}^{2}\right)$ is obtained in comparison to other deep-inelastic analyses. At a given scale $Q^{2}$ the Mellin moments of the parton distribution functions can be calculated under some assumptions on their extrapolation outside the measured region towards small and very large values of the Bjorken variable $x$. We also analyze, to which extend the present data contain higher twist contributions.

The paper is organized as follows. In Section 2 the basic formalism is lined out. The data analysis is described in Section 3. In Section 4 details of the fit are given and Section 5 deals with the error analysis. The results of the QCD analysis are presented in Section 6. In Section 7 we determine potential higher twist contributions and Section 8 contains the conclusions. In Appendix A we describe the FORTRAN-code through which the polarized parton distributions and structure functions can be obtained for numerical analyses.

\section{Basic formalism}

The twist- 2 contributions to the spin-dependent structure function $g_{1}\left(x, Q^{2}\right)$ are given in terms of a Mellin convolution of the polarized singlet $\Delta \Sigma$, the gluon $\Delta G$ and the flavor nonsinglet (NS) $\Delta q_{i}^{\mathrm{NS}}$ densities with the corresponding Wilson coefficient functions $\Delta C_{i}^{A}$ by

$$
\begin{aligned}
g_{1}\left(x, Q^{2}\right)= & \frac{1}{2} \sum_{i=1}^{N_{f}} e_{i}^{2} \int_{x}^{1} \frac{d z}{z}\left[\frac{1}{N_{f}} \Delta \Sigma\left(\frac{x}{z}, \mu_{f}^{2}\right) \Delta C_{q}^{S}\left(z, \frac{Q^{2}}{\mu_{f}^{2}}\right)\right. \\
& \left.+\Delta G\left(\frac{x}{z}, \mu_{f}^{2}\right) \Delta C_{G}\left(z, \frac{Q^{2}}{\mu_{f}^{2}}, \frac{m_{c}^{2}}{Q^{2}}\right)+\Delta q_{i}^{\mathrm{NS}}\left(\frac{x}{z}, \mu_{f}^{2}\right) \Delta C_{q}^{\mathrm{NS}}\left(z, \frac{Q^{2}}{\mu_{f}^{2}}\right)\right]
\end{aligned}
$$

Here $x$ is the Bjorken variable, $e_{i}$ denotes the charge of the $i$ th quark flavor in units of the elementary charge and $N_{f}$ is the number of light flavors. The scale $\mu_{f}$ denotes the factorization scale which is introduced to absorb the collinear singularities into the renormalized partonic

\footnotetext{
1 Twist-3 contributions are connected by target mass effects, cf. [16].
} 
distribution functions. In addition to the factorization scale there is the renormalization scale $\mu_{r}$ of the strong coupling constant $\alpha_{s}\left(\mu_{r}^{2}\right)$. The gluonic Wilson coefficient $\Delta C_{G}$ accounts for the massless as well the massive contributions due to charm quark production for $W^{2}>\left(2 m_{c}+\right.$ $\left.m_{N}\right)^{2}$ with $m_{c}=1.5 \mathrm{GeV}$ [32], at first order. ${ }^{2}$ For the implementation of the Wilson coefficients in Mellin space we refer to [34]. The parton densities and the Wilson coefficient functions are dependent on these scales and obey corresponding renormalization group equations, while the structure function $g_{1}\left(x, Q^{2}\right)$, as a physical observable, is independent of the choice of both scales $\mu_{f}^{2}$ and $\mu_{r}^{2}$.

The polarized singlet and non-singlet parton densities which occur in Eq. (1) are expressed by the individual quark flavor contributions as

$$
\begin{aligned}
& \Delta \Sigma\left(z, \mu_{f}^{2}\right)=\sum_{i=1}^{N_{f}}\left[\Delta q_{i}\left(z, \mu_{f}^{2}\right)+\Delta \bar{q}_{i}\left(z, \mu_{f}^{2}\right)\right] \\
& \Delta q_{i}^{\mathrm{NS}}\left(z, \mu_{f}^{2}\right)=\Delta q_{i}\left(z, \mu_{f}^{2}\right)+\Delta \bar{q}_{i}\left(z, \mu_{f}^{2}\right)-\frac{1}{N_{f}} \Delta \Sigma\left(z, \mu_{f}^{2}\right),
\end{aligned}
$$

where $\Delta q_{i}$ denotes the polarized quark distribution of the $i$ th light flavor. We will consider three light quark flavors in the present analysis and treat charm quark production through the gluon fusion process in leading order [32] in the fixed flavor number scheme for partons. As well known, the following flavor non-singlet combinations contribute in the case of pure photon exchange considering proton and neutron targets,

$$
\begin{aligned}
& \Delta_{p}^{\mathrm{NS}}+\Delta_{n}^{\mathrm{NS}}=\frac{1}{9}[(\Delta u+\Delta \bar{u})+(\Delta d+\Delta \bar{d})]-\frac{2}{9}(\Delta s+\Delta \bar{s}), \\
& \Delta_{p}^{\mathrm{NS}}-\Delta_{n}^{\mathrm{NS}}=\frac{1}{3}[(\Delta u+\Delta \bar{u})-(\Delta d+\Delta \bar{d})] .
\end{aligned}
$$

As usual one assumes

$$
\frac{1}{6} \Delta q_{\mathrm{sea}}=\Delta \bar{q}_{i}, \quad i=u, d, s,
$$

although later highest precision measurements could even reveal a breaking of this relation. The distribution

$$
\Delta \bar{d}-\Delta \bar{u}
$$

cannot be measured in DIS data, whereas polarized Drell-Yan data are well suited for its determination, cf. e.g. [31]. For the measurement of the polarized strangeness distribution function one would wish to have sufficiently precise polarized di-muon samples available. Nonetheless first flavor separations have been attempted in performing SIDIS analyses, cf. [25].

The running coupling constant $a_{s}=\alpha_{s} /(4 \pi)$ is obtained as the solution of

$$
\frac{d a_{s}\left(\mu_{r}^{2}\right)}{d \log \left(\mu_{r}^{2}\right)}=-\beta_{0} a_{s}^{2}\left(\mu_{r}^{2}\right)-\beta_{1} a_{s}^{3}\left(\mu_{r}^{2}\right)+O\left(a_{s}^{4}\right),
$$

where, in the $\overline{\mathrm{MS}}$-scheme, the coefficients of the $\beta$-function are given by

\footnotetext{
2 2nd order corrections were calculated in the asymptotic range $Q^{2} \gg m^{2}$ in Ref. [33].
} 


$$
\begin{aligned}
& \beta_{0}=\frac{11}{3} C_{A}-\frac{4}{3} T_{F} N_{f}, \\
& \beta_{1}=\frac{34}{3} C_{A}^{2}-\frac{20}{3} C_{A} T_{F} N_{f}-4 C_{F} T_{F} N_{f},
\end{aligned}
$$

with the color factors $C_{A}=3, T_{F}=1 / 2$, and $C_{F}=4 / 3$. The matching of the scale $\Lambda_{\mathrm{QCD}}^{N_{f}}$ is performed at $Q^{2}=m_{c}^{2}, m_{b}^{2}$, with $m_{c}=1.5 \mathrm{GeV}$ and $m_{b}=4.5 \mathrm{GeV}$.

In the present analysis the spin-dependent structure functions $g_{1}^{p}\left(x, Q^{2}\right)$ and $g_{1}^{n}\left(x, Q^{2}\right)$ will be considered referring to $N_{f}=3$ light partonic flavors, i.e. $i=u, d, s$. The spin-dependent structure function $g_{1}^{d}\left(x, Q^{2}\right)$ is represented in terms of $g_{1}^{p}\left(x, Q^{2}\right)$ and $g_{1}^{n}\left(x, Q^{2}\right)$ using the relation

$$
g_{1}^{d}\left(x, Q^{2}\right)=\frac{1}{2}\left(1-\frac{3}{2} \omega_{D}\right)\left[g_{1}^{p}\left(x, Q^{2}\right)+g_{1}^{n}\left(x, Q^{2}\right)\right],
$$

where $\omega_{D}=0.05 \pm 0.01$ is the $D$-state wave probability for the deuteron [35].

The change of the parton densities with respect to the factorization scale $\mu_{f}^{2}=Q^{2}$ is described by the evolution equations, which read

$$
\begin{aligned}
& \frac{\partial \Delta q_{i}^{\mathrm{NS}}\left(x, Q^{2}\right)}{\partial \log Q^{2}}=\Delta P_{q q}^{\mathrm{NS}}\left(x, a_{s}\right) \otimes \Delta q_{i}^{\mathrm{NS}}\left(x, Q^{2}\right), \\
& \frac{\partial}{\partial \log Q^{2}}\left(\begin{array}{c}
\Delta \Sigma\left(x, Q^{2}\right) \\
\Delta G\left(x, Q^{2}\right)
\end{array}\right)=\Delta \boldsymbol{P}\left(x, a_{s}\right) \otimes\left(\begin{array}{c}
\Delta \Sigma\left(x, Q^{2}\right) \\
\Delta G\left(x, Q^{2}\right)
\end{array}\right),
\end{aligned}
$$

with

$$
\Delta \boldsymbol{P}\left(x, a_{s}\right) \equiv\left(\begin{array}{cc}
\Delta P_{q q}\left(x, a_{s}\right) & 2 N_{f} \Delta P_{q g}\left(x, a_{s}\right) \\
\Delta P_{g q}\left(x, a_{s}\right) & \Delta P_{g g}\left(x, a_{s}\right)
\end{array}\right) .
$$

The symbol $\otimes$ denotes the Mellin convolution

$$
[A \otimes B](x)=\int_{0}^{1} d x_{1} d x_{2} \delta\left(x-x_{1} x_{2}\right) A\left(x_{1}\right) B\left(x_{2}\right) .
$$

The spin-dependent coefficient functions $\Delta C_{i}^{A}$ and anomalous dimensions $\Delta P_{i j}$ are calculated to next-to-leading order in the $\overline{\mathrm{MS}}$-scheme [36-38], which we use in the present analysis. As seen from Eqs. (11) and (12), the flavor non-singlet densities $\Delta q_{i}^{\mathrm{NS}}$ evolve independently, while $\Delta \Sigma$ and $\Delta G$ are coupled in the evolution.

In order to solve the evolution equations, a Mellin transformation of Eqs. (11), (12) and the polarized parton densities $\Delta f(x)$ is being performed by calculating its $N$ th Mellin moment:

$$
\mathbf{M}[\Delta f](N)=\int_{0}^{1} d x x^{N-1} \Delta f(x), \quad N \geqslant N_{0}, N \in \mathbf{R} .
$$

Here $N_{0}$ is chosen such that the integral (15) converges. Under this transformation the Mellin convolution $\otimes$ turns into an ordinary product. After the transformation has been performed the $\operatorname{argument} N$ is analytically continued to the complex plane. This also requires analytic continuations of harmonic sums [39], which is outlined in Ref. [40] in detail. The fundamental method of solving Eqs. (11) and (12) is described in the literature in detail, see e.g. Refs. [37,41,42]. 
To next-to-leading order (NLO) the solution of the flavor non-singlet and singlet evolution equations are given by

$$
\begin{aligned}
& \Delta q_{i}^{\mathrm{NS}}\left(N, a_{s}\right)=\left(\frac{a_{s}}{a_{0}}\right)^{-P_{\mathrm{NS}}^{(0)} / \beta_{0}}\left[1-\frac{1}{\beta_{0}}\left(a_{s}-a_{0}\right)\left(\Delta P_{\mathrm{NS}}^{(1)}-\frac{\beta_{1}}{\beta_{0}} P_{\mathrm{NS}}^{(0)}\right)\right] \Delta q_{i}^{\mathrm{NS}}\left(N, a_{0}\right), \\
& \left(\begin{array}{c}
\Delta \Sigma\left(N, a_{s}\right) \\
\Delta G\left(N, a_{s}\right)
\end{array}\right)=\left[\mathbf{1}+a_{s} \boldsymbol{U}_{1}(N)\right] \boldsymbol{L}\left(N, a_{s}, a_{0}\right)\left[\mathbf{1}-a_{0} \boldsymbol{U}_{1}(N)\right]\left(\begin{array}{c}
\Delta \Sigma\left(N, a_{0}\right) \\
\Delta G\left(N, a_{0}\right)
\end{array}\right) .
\end{aligned}
$$

Here $P_{\mathrm{NS}}^{(0)}$ is the leading order splitting function for the quark-quark transition. $\Delta P_{\mathrm{NS}}^{(1)}$ denotes the NLO non-singlet ' - ' splitting function, $a_{s} \equiv a_{s}\left(Q^{2}\right)$ and $a_{0}=a_{s}\left(Q_{0}^{2}\right)$, with $Q_{0}^{2}$ being the input scale. The matrices $\boldsymbol{U}_{1}$ and $\boldsymbol{L}$ are evolution matrices, for details see Ref. [42]. We refrain from applying so-called small $x$ resummations $[43,44]$, since they are very sensitive to several series of less singular terms $[42,43]$, which are yet unknown. Furthermore, no factorization theorem exists for these terms through which non-perturbative and perturbative contributions can be separated in a well defined way. Likewise, no other evolution equation than that governing mass singularities exists to deal with these terms.

Due to the factor-structure in Eqs. (16) and (17), the Gaussian error propagation of the input density parameters can be calculated analytically, cf. [23], for the whole $Q^{2}$ region. The covariance matrix of the parton distributions alone is completely determined by the fit to the data at the input scale.

The input distributions $\Delta_{p, n}^{\mathrm{NS}}\left(N, a_{0}\right), \Delta \Sigma\left(N, a_{0}\right)$ and $\Delta G\left(N, a_{0}\right)$ are evolved to the scale $Q^{2}$. An inverse Mellin-transform to $x$-space is then performed by a contour integral in the complex plane around all singularities on the real axis for $x \leqslant x_{0} \leqslant 1$, which can be written as

$$
\Delta f(x)=\frac{1}{\pi} \int_{0}^{\infty} d z \operatorname{Im}\left[\exp (i \phi) x^{-c(z)} \Delta f[c(z)]\right] .
$$

In practice an integral along the path $c(z)=c_{1}+\rho[\cos (\phi)+i \sin (\phi)]$, with $c_{1}=1.1, \rho \geqslant 0$ and $\phi=(3 / 4) \pi$ is performed. The upper bound on $\rho$ has to be chosen in accordance with the numerical convergence of the integral (18) in practice. The result $\Delta f(x)$ for the respective distribution depends on the parameters of the spin-dependent parton distributions chosen at the input scale $Q_{0}^{2}$, which are determined by a fit to the data and to $\Lambda_{\mathrm{QCD}}$ or $\alpha_{S}\left(M_{Z}^{2}\right)$, respectively.

\section{Data analysis}

The QCD analysis being performed in the following is based on the spin-dependent structure functions $g_{1}^{p, d, n}\left(x, Q^{2}\right)$. These structure functions are extracted from the experimental cross section asymmetries for longitudinally polarized leptons scattered off longitudinally polarized nucleons,

$$
A_{\|}=\frac{\sigma^{\rightrightarrows}-\sigma^{\rightleftarrows}}{\sigma \vec{\rightleftarrows}+\sigma^{\rightleftarrows}} .
$$

The arrows $\rightrightarrows(\rightleftarrows)$ denote parallel (anti-parallel) relative spin orientation of the incoming lepton and nucleon. The structure function ratio $g_{1} / F_{1}$ and the longitudinal virtual-photon asymmetry $A_{1}$ are related to $A_{\|}$by

$$
\frac{g_{1}}{F_{1}}=\frac{1}{\left(1+\gamma^{2}\right)}\left[\frac{A_{\|}}{D}+(\gamma-\eta) A_{2}\right]
$$


and

$$
A_{1}=\frac{A_{\|}}{D}-\eta A_{2},
$$

with

$$
\frac{g_{1}}{F_{1}}=\frac{1}{\left(1+\gamma^{2}\right)}\left[A_{1}+\gamma A_{2}\right] .
$$

The asymmetry $A_{2}$ is the transverse virtual-photon asymmetry and constitutes only a small correction to $g_{1}$. Its contribution has been treated differently by various experiments as will be discussed below. The other variables are given by

$$
\begin{aligned}
& D=\frac{1-(1-y) \epsilon}{1+\epsilon R\left(x, Q^{2}\right)}, \\
& \gamma=\frac{2 M x}{\sqrt{Q^{2}}}, \\
& \eta=\frac{\epsilon \gamma y}{1-\epsilon(1-y)}, \\
& \epsilon=\frac{4(1-y)-\gamma^{2} y^{2}}{2 y^{2}+4(1-y)+\gamma^{2} y^{2}} .
\end{aligned}
$$

Here $D$ denotes the virtual photon depolarization factor. It determines the fraction of the incoming lepton polarization transferred to the virtual photon. The variables $\epsilon, \gamma$ and $\eta$ are kinematic factors, $M$ denotes the mass of the nucleon and $y=\left(E-E^{\prime}\right) / E$ is a Bjorken scaling variable which describes the normalized energy transfer to the virtual photon, with $E$ the incoming energy and $E^{\prime}$ the energy of the scattered lepton in the target rest frame. Finally, $R$ denotes the ratio of the longitudinal and transverse virtual-photon absorption cross section $R\left(x, Q^{2}\right)=\sigma_{L} / \sigma_{T}$, which is experimentally well determined in the kinematic region considered in the present analysis.

In order to obtain $g_{1}\left(x, Q^{2}\right)$ the measured ratio $g_{1} / F_{1}$ has to be multiplied by the spinindependent structure function $F_{1}\left(x, Q^{2}\right)$ :

$$
g_{1}\left(x, Q^{2}\right)=\left(\frac{g_{1}}{F_{1}}\right)\left(x, Q^{2}\right) \times F_{1}\left(x, Q^{2}\right) .
$$

The structure function $F_{1}\left(x, Q^{2}\right)$ can be calculated from the structure function $F_{2}\left(x, Q^{2}\right)$ by

$$
F_{1}\left(x, Q^{2}\right)=\frac{\left(1+\gamma^{2}\right)}{2 x\left(1+R\left(x, Q^{2}\right)\right)} F_{2}\left(x, Q^{2}\right) .
$$

For $R\left(x, Q^{2}\right)$ and $F_{2}\left(x, Q^{2}\right)$ parameterizations of existing measurements are available as will be discussed below.

The following data sets have been used in the present analysis: the EMC proton data [1], the E142 neutron data [2], the HERMES neutron data [3], the E154 neutron data [4,15], the SMC proton and deuteron data [5], the E143 proton and deuteron data [6], the HERMES reanalyzed proton and the new deuteron data [7], the E155 deuteron data [8], the E155 proton data [9], the COMPASS deuteron data [11], the JLAB neutron [10], proton and deuteron data [12, 13], and the COMPASS proton data [14]. ${ }^{3}$ The number of data points with $Q^{2}>1.0 \mathrm{GeV}^{2}$ and

\footnotetext{
3 Earlier data from Ref. [45] are not considered.
} 
$W^{2}>3.24 \mathrm{GeV}^{2}$ from the different data sets are summarized in Table 1 together with the $x$ and $Q^{2}$ ranges of the different experiments. In order to obtain the best possible statistical accuracy data on $A_{1}, g_{1} / F_{1}$ and $g_{1}$ are not averaged over the different $Q^{2}$ values measured within a certain $x$ bin. In total 1385 data points are used. Using $A_{1}$ and $g_{1} / F_{1}$ data has, in addition to the higher statistics, the advantage of calculating $g_{1}$ for all these data sets in a unique way. Furthermore, $g_{1}$ data are sometimes only published as obtained from the average of asymmetries measured at different $Q^{2}$ values, while for the QCD analysis it is important to maintain the $Q^{2}$ dependence of the measured quantities.

The SLAC parameterization $R_{1990}$ [46] is used by most of the experiments when extracting $g_{1}$. At the time of the EMC experiment this parameterization was not available yet and $R$ was assumed to be $Q^{2}$ independent. SMC adopted a combination of $R_{1990}$ (for $x>0.12$ ) and a parameterization derived by NMC [47] (for $x<0.12$ ). In the E155 experiment a recent SLAC parameterization for $R, R(1998)$ [48], was used. The changes in the data caused by using the different $R$-parameterizations, however, are not significant and stay within the experimental errors. ${ }^{4}$ For all $A_{1}$ and $g_{1} / F_{1}$ data sets entering the present QCD analysis the $\operatorname{SLAC} R_{1990}\left(x, Q^{2}\right)$ [46] and the NMC $F_{2}\left(x, Q^{2}\right)$-parameterization [49] is used to perform the calculation of $g_{1}$. The same parameterizations were used by the E154 experiment while JLAB applied the $R(1998)$ SLAC parameterization of $R$.

The magnitude of $A_{2}$ has been measured by SMC [50], E154 [51], E143 [6], E155x [52] and JLAB [10] and was found to be small. Its contribution to $g_{1} / F_{1}$ and $A_{1}$ is further suppressed by the kinematic factors $\gamma$ and $\eta$ and could in principle be neglected to a good approximation. On the other hand all the measurements have shown that the $A_{2}$ contribution can be approximated by the Wandzura-Wilczek expression [53], which is calculated from the spin-dependent structure function $g_{1}\left(x, Q^{2}\right)$ assuming that twist-2 contributions are dominant according to 5

$$
\begin{aligned}
A_{2}\left(x, Q^{2}\right) & =\frac{\gamma\left(x, Q^{2}\right)}{F_{1}\left(x, Q^{2}\right)}\left[g_{1}\left(x, Q^{2}\right)+g_{2}\left(x, Q^{2}\right)\right] \\
& \stackrel{W W}{\simeq} \frac{\gamma\left(x, Q^{2}\right)}{F_{1}\left(x, Q^{2}\right)} \int_{x}^{1} \frac{d z}{z} g_{1}\left(z, Q^{2}\right) .
\end{aligned}
$$

The E143 experiment has exploited its measurement of $A_{2}$ at $29.1 \mathrm{GeV}$ and used the WandzuraWilczek expression to account for $A_{2}$ for the other two lower beam energies. The measurement of $A_{2}$ by E154 and E155x was done after having published the data on $A_{1}$ and was therefore not available for a $A_{2}$ correction of $A_{1}$. While E154 neglected $A_{2}$, the E155 experiment has used the Wandzura-Wilczek approximation throughout its data. The JLAB measurement of $A_{2}$ went into the extraction of $g_{1}^{n}$. In HERMES the $A_{2}$ contribution to $g_{1}^{p, d} / F_{1}^{p, d}$ data has been accounted for by using a parameterization for $A_{2}$ obtained from a fit $A_{2}=C M_{p} x / \sqrt{Q^{2}}$ to all available proton and deuteron data [7]. For all $A_{1}$ data sets used in this analysis $g_{1}$ has been calculated with the application of the Wandzura-Wilczek correction for $A_{2}$.

The target mass corrections to the structure function $g_{1}\left(x, Q^{2}\right)$ are given by $[16,59]$

\footnotetext{
4 The EMC proton data, where the biggest impact is expected, change by a few percent only, see Ref. [18].

5 Note that this relation holds also in the presence of quark and target mass corrections [16,54,55], for non-forward scattering [56], for diffractive scattering [57], and the gluonic contributions to heavy flavor production [58]. Related integral relations for twist-3 contributions and structure functions with electro-weak couplings were derived in Refs. [16, $55]$.
} 
Table 1

Number of data points on $A_{1}, g_{1} / F_{1}$ or $g_{1}$ for $Q^{2}>1.0 \mathrm{GeV}^{2}$ and $W^{2}>3.24 \mathrm{GeV}^{2}$ used in the present QCD analysis. For each experiment are given the $x$ and $Q^{2}$ ranges, the type of quantity measured, the number of data points for each given target, and the fitted normalization shifts $\mathcal{N}_{i}$ (see text).

\begin{tabular}{|c|c|c|c|c|c|}
\hline \multirow[t]{2}{*}{ Experiment } & \multirow[t]{2}{*}{$x$-range } & \multirow{2}{*}{$\begin{array}{l}Q^{2} \text {-range } \\
{\left[\mathrm{GeV}^{2}\right]}\end{array}$} & \multicolumn{2}{|c|}{ Data points } & \multirow[t]{2}{*}{$\mathcal{N}_{i}$} \\
\hline & & & Type & $\#$ & \\
\hline E143(p) [6] & $0.027-0.749$ & $1.17-9.52$ & $g_{1} / F_{1}$ & 82 & 0.963 \\
\hline $\operatorname{HERMES}(p)$ [7] & $0.026-0.731$ & $1.12-14.29$ & $A_{1}$ & 37 & 0.970 \\
\hline E155(p) [9] & $0.015-0.750$ & $1.22-34.72$ & $g_{1} / F_{1}$ & 24 & 1.003 \\
\hline SMC(p) [5] & $0.004-0.484$ & $1.14-72.10$ & $A_{1}$ & 59 & 0.960 \\
\hline $\operatorname{EMC}(p)[1]$ & $0.015-0.466$ & $3.50-29.5$ & $A_{1}$ & 10 & 0.964 \\
\hline CLAS1(p) [12] & $0.125-0.575$ & $1.10-4.16$ & $A_{1}$ & 10 & 1.010 \\
\hline CLAS2(p) [13] & $0.292-0.592$ & $1.01-4.96$ & $g_{1} / F_{1}$ & 191 & 1.030 \\
\hline $\operatorname{COMPASS}(p)$ [14] & $0.005-0.568$ & $1.10-62.10$ & $A_{1}$ & 15 & 0.955 \\
\hline \multicolumn{3}{|l|}{ Proton } & \multicolumn{3}{|c|}{428} \\
\hline E143(d) [6] & $0.027-0.749$ & $1.17-9.52$ & $g_{1} / F_{1}$ & 82 & 0.960 \\
\hline HERMES(d) [7] & $0.026-0.731$ & $1.12-14.29$ & $A_{1}$ & 37 & 0.970 \\
\hline E155(d) $[8]$ & $0.015-0.750$ & $1.22-34.79$ & $g_{1} / F_{1}$ & 24 & 0.979 \\
\hline $\mathrm{SMC}(\mathrm{d})[5]$ & $0.004-0.483$ & $1.14-71.76$ & $A_{1}$ & 65 & 0.998 \\
\hline COMPASS(d) [11] & $0.005-0.566$ & $1.10-55.30$ & $A_{1}$ & 15 & 0.952 \\
\hline CLAS1(d) [12] & $0.125-0.575$ & $1.01-4.16$ & $A_{1}$ & 10 & 1.003 \\
\hline CLAS2(d) [13] & $0.298-0.636$ & $1.01-4.16$ & $g_{1} / F_{1}$ & 662 & 1.014 \\
\hline \multicolumn{3}{|l|}{ Deuteron } & \multicolumn{3}{|c|}{895} \\
\hline E142(n) [2] & $0.035-0.466$ & $1.10-5.50$ & $A_{1}$ & 33 & 0.989 \\
\hline HERMES(n) [3] & $0.033-0.464$ & $1.22-5.25$ & $g_{1}$ & 9 & 0.970 \\
\hline E154(n) [4]/[15] & $0.017-0.564$ & $1.20-15.00$ & $g_{1}$ & 17 & 0.980 \\
\hline JLAB(n) [10] & $0.330-0.600$ & $2.71-4.83$ & $g_{1}$ & 3 & 1.000 \\
\hline \multicolumn{3}{|l|}{ Neutron } & \multicolumn{3}{|c|}{62} \\
\hline \multicolumn{3}{|l|}{ Total } & \multicolumn{3}{|c|}{1385} \\
\hline
\end{tabular}

$$
\begin{aligned}
g_{1}^{\mathrm{TM}}\left(x, Q^{2}\right)= & \frac{x}{\xi} \frac{g_{1}\left(\xi, Q^{2}\right)}{\left(1+4 M^{2} x^{2} / Q^{2}\right)^{3 / 2}}+\frac{4 M^{2} x^{2}}{Q^{2}} \frac{x+\xi}{\xi\left(1+4 M^{2} x^{2} / Q^{2}\right)^{2}} \int_{\xi}^{1} \frac{d \xi_{1}}{\xi_{1}} g_{1}\left(\xi_{1}, Q^{2}\right) \\
& -\frac{4 M^{2} x^{2}}{Q^{2}} \frac{2-4 M^{2} x^{2} / Q^{2}}{2\left(1+4 M^{2} x^{2} / Q^{2}\right)^{5 / 2}} \int_{\xi}^{1} \frac{d \xi_{1}}{\xi_{1}} \int_{\xi_{1}}^{1} \frac{d \xi_{2}}{\xi_{2}} g_{1}\left(\xi_{2}, Q^{2}\right) .
\end{aligned}
$$

Here $M$ denotes the nucleon mass and

$$
\xi=\frac{2 x}{1+\left(1+4 M^{2} x^{2} / Q^{2}\right)^{1 / 2}}
$$

is the Nachtmann variable. We refrain to use approximations to (30) in terms of power series in $M^{2} / Q^{2}$ since the convergence of the corresponding series is problematic as being lined out in Ref. [16].

The data sets contain both statistical and systematic errors. It is known that the systematic errors are partly correlated, which would lead to an overestimation of the errors when added in quadrature with the statistical ones, and hence to a reduction of the $\chi^{2}$ value in the fitting procedure. To treat all data sets on the same footing only statistical errors were used. However, 
a relative normalization shift, $\mathcal{N}_{i}$, between the different data sets was allowed within the normalization uncertainties, $\Delta \mathcal{N}_{i}$, quoted by the experiments. These normalization shifts were fitted once and then fixed, see Table 1 . Thereby the main systematic uncertainties coming from the measurements of the luminosity and the beam and target polarization were taken into account. The normalization shift for each data set enters as an additional term in the $\chi^{2}$-expression for the fit which then reads

$$
\chi^{2}=\sum_{i=1}^{n^{e x p}}\left[\frac{\left(\mathcal{N}_{i}-1\right)^{2}}{\left(\Delta \mathcal{N}_{i}\right)^{2}}+\sum_{h, k=1}^{n^{\text {data }}}\left(\mathcal{N}_{i} g_{1 i, h}^{\text {data }}-g_{1, h}^{\text {theor }}\right)\left(\mathcal{C}_{i}^{-1}\right)^{h k}\left(\mathcal{N}_{i} g_{1 i, k}^{\text {data }}-g_{1, k}^{\text {theor }}\right)\right],
$$

where the sums run over all data sets and in each data set over all data points. The covariance matrices $\mathcal{C}_{i}$ are diagonal for each experiment except for the case of the HERMES data [7]. The statistical errors, and consequently the covariance matrices $\mathcal{C}_{i}$, have been rescaled by the normalization factors $\mathcal{N}_{i}$. The minimization of the $\chi^{2}$ value above to determine the best parameterization of the polarized parton distributions is performed using the program MINUIT [60]. Only fits giving a positive definite covariance matrix at the end have been accepted in order to be able to calculate the fully correlated $1 \sigma$ statistical error bands.

\section{Parameterization of the polarized parton distributions}

The shape chosen for the parameterization of the polarized parton distributions $\Delta f_{i}\left(x, Q^{2}\right)$ in $x$-space at the input scale $Q_{0}^{2}$ is:

$$
x \Delta f_{i}\left(x, Q_{0}^{2}\right)=\eta_{i} A_{i} x^{a_{i}}(1-x)^{b_{i}}\left(1+\rho_{i} x^{\frac{1}{2}}+\gamma_{i} x\right) .
$$

The normalization constant $A_{i}$, being given by

$$
A_{i}^{-1}=\left(1+\gamma_{i} \frac{a_{i}}{a_{i}+b_{i}+1}\right) B\left(a_{i}, b_{i}+1\right)+\rho_{i} B\left(a_{i}+\frac{1}{2}, b_{i}+1\right),
$$

is calculated such that

$$
\eta_{i}=\int_{0}^{1} d x \Delta f_{i}\left(x, Q_{0}^{2}\right)
$$

is the first moment of $\Delta f_{i}\left(x, Q_{0}^{2}\right)$ at the input scale. Here, $B(a, b)$ is the Euler Beta-function being related to the $\Gamma$-function by $B(a, b)=\Gamma(a) \Gamma(b) / \Gamma(a+b)$. The choice of the shape (32) is applied in various QCD analyses of unpolarized data, see e.g. Ref. [61]. The term $x^{a_{i}}$ controls the behavior of the parton density at low and $(1-x)^{b_{i}}$ that at large values of $x$, respectively. The remaining polynomial factor accounts for potential degrees of freedom at medium $x$. At the same time not too many parameters can be sufficiently constrained by the data available at present. In particular the parameters $\rho_{i}$, which play a role in unpolarized analyses, [28], are set to zero, cf. also $[23,25]$.

If the QCD evolution equations are solved in Mellin space as described in Section 2 a Mellin transformation of the polarized parton density $\Delta f\left(x, Q^{2}\right)$ is performed for complex arguments $N$ : 


$$
\begin{aligned}
\mathbf{M}\left[\Delta f_{i}\left(x, Q_{0}^{2}\right)\right](N) & =\int_{0}^{1} x^{N-1} d x \Delta f_{i}\left(x, Q_{0}^{2}\right) \\
& =\eta_{i} A_{i}\left(1+\gamma_{i} \frac{N-1+a_{i}}{N+a_{i}+b_{i}}\right) B\left(N-1+a_{i}, b_{i}+1\right) .
\end{aligned}
$$

In the present analysis we assume (approximate) SU(3) flavor symmetry the sea quark distribution is given by

$$
\begin{aligned}
& \Delta \bar{q}_{s}\left(x, Q^{2}\right)=\Delta \bar{u}\left(x, Q^{2}\right)=\Delta \bar{d}\left(x, Q^{2}\right)=\Delta s\left(x, Q^{2}\right)=\Delta \bar{s}\left(x, Q^{2}\right), \\
& \Delta \Sigma\left(x, Q^{2}\right)=\Delta u_{v}\left(x, Q^{2}\right)+\Delta d_{v}\left(x, Q^{2}\right)+6 \Delta \bar{q}_{s}\left(x, Q^{2}\right) .
\end{aligned}
$$

We refer to the inclusive polarized DIS World Data only. A breaking of the flavor symmetry also for the light (sea) quarks in the polarized case is probable and has been clearly observed in the unpolarized case, cf. e.g. [31]. In the polarized case first attempts have been made to determine the individual sea quark distributions, cf. Refs. $[25,62]$. We consider the evolution of the complete light polarized sea.

Under the above assumption four spin-dependent parton densities have to be determined in the QCD analysis. They are chosen to be: $\Delta u_{v}\left(x, Q^{2}\right), \Delta d_{v}\left(x, Q^{2}\right), \Delta \bar{q}_{s}\left(x, Q^{2}\right)$ and $\Delta G\left(x, Q^{2}\right)$. As seen from Eq. (32), each spin-dependent density contains five parameters which gives a total of 20 for all four. It has been found that, in order to meet the quality of the available data and the reliability of the fitting procedure, this large number of free parameters has to be reduced, which is discussed below.

The first moments of the polarized valence distributions $\Delta u_{v}$ and $\Delta d_{v}, \eta_{u_{v}}$ and $\eta_{d_{v}}$, can be fixed exploiting the knowledge of the parameters $F$ and $D$ as measured in neutron and hyperon $\beta$-decays according to the relations:

$$
\begin{aligned}
& \eta_{u_{v}}-\eta_{d_{v}}=F+D, \\
& \eta_{u_{v}}+\eta_{d_{v}}=3 F-D .
\end{aligned}
$$

A re-evaluation of $F$ and $D$ was performed on the basis of updated $\beta$-decay constants [63] leading to

$$
F=0.464 \pm 0.008 \text { and } D=0.806 \pm 0.008,
$$

and consequently to

$$
\eta_{u_{v}}=0.928 \pm 0.014 \text { and } \eta_{d_{v}}=-0.342 \pm 0.018 \text {. }
$$

In order to compensate for the present insufficient accuracy of the data, a certain number of parameters is set to zero after this was thoroughly suggested by initial fits. This applies to $\rho_{u_{v}}=\rho_{d_{v}}=0, \gamma_{\bar{q}_{s}}=\rho_{\bar{q}_{s}}=0$, and $\gamma_{G}=\rho_{G}=0$. The number of parameters to be fitted for each polarized parton density is reduced to three, i.e. to 12 in total. In addition the QCD scale $\Lambda_{\mathrm{QCD}}$ is fitted.

In the analysis it turns out that the four parameters $\gamma_{u_{v}}, \gamma_{d_{v}}, b_{\bar{q}_{s}}$, and $b_{G}$ have very large uncertainties. The precision of the data is not high enough to constrain these parameters sufficiently. Altering them within these uncertainties does not lead to a significant change of $\chi^{2}$. These four parameters were therefore fixed. The first two of them were fixed at their values obtained in the initial fitting pass, $\gamma_{u_{v}}=27.64$ and $\gamma_{d_{v}}=44.26$. In fixing the high- $x$ slopes $b_{G}$ and $b_{\bar{q}_{s}}$ a relation was adopted as derived from the unpolarized parton densities, 
$b_{\bar{q}_{s}} / b_{G}(\mathrm{pol})=b_{\bar{q}_{s}} / b_{G}($ unpol $)=1.44$. Fitting with this constraint led to the following choice: $b_{G}=5.61$ and $b_{\bar{q}_{s}}=8.08$, see e.g. Ref. [64].

A second relation was adopted to constrain the low- $x$ behavior of the spin-dependent gluon density with respect to the low- $x$ behavior of the spin-dependent sea-quark distribution by $a_{G}=$ $a_{\bar{q}_{s}}+C$ with $C=1$. This relation, together with the relation for the high- $x$ slopes, are suited to establish positivity for $\Delta G$ and $\Delta \bar{q}_{s}$. No explicit positivity constraint has been enforced for $\Delta u_{v}$ and $\Delta d_{v}$.

\section{Determination of the errors}

\subsection{Calculation of statistical errors}

The evolved polarized parton densities and structure functions are functions of the input densities. Let $\Delta f\left(x, Q^{2} ;\left.p_{i}\right|_{i=1, k}\right)$ be the evolved polarized density at the scale $Q^{2}$ depending on the parameters $\left.p_{i}\right|_{i=1, k}$. Then its correlated statistical error as given by Gaussian error propagation is

$$
(\sigma \Delta f)^{2}=\sum_{i, j=1}^{k}\left(\frac{\partial \Delta f}{\partial p_{i}} \frac{\partial \Delta f}{\partial p_{j}}\right) \operatorname{cov}\left(p_{i}, p_{j}\right),
$$

where $\operatorname{cov}\left(p_{i}, p_{j}\right)$ are the elements of the covariance matrix determined in the QCD analysis. The gradients $\partial \Delta f / \partial p_{i}$ at the input scale $Q_{0}^{2}$ can be calculated analytically. Their values at $Q^{2}$ are evaluated through the evolution and can then be used to compute the errors according to Eq. (42). As shown in Section 2, the covariance matrix is completely determined by the fit at the input scale and does not change when the evolution is done in Mellin space. That means it can be used at any scale $Q^{2}$. For the expressions of the gradients at the input scale see Ref. [23].

Apart from statistical errors the data are also subject to systematic uncertainties which are even partly correlated. These correlations are not published by the experiments and can hence not be taken into account here. In the following the influence of experimental and theoretical systematic uncertainties will be investigated.

\subsection{Determination of experimental systematic uncertainties}

The experimental systematic uncertainties were estimated from the following sources:

1. The variation of the data within their experimental systematic uncertainties.

The procedure used to obtain the contribution from the experimental systematic uncertainties consists in shifting each data set by $\pm \sigma_{\text {syst }}$ while leaving the other data sets at their central values and looking at how much the polarized distributions change. The extreme changes from the 'central' distribution were taken as the systematic uncertainties. This was done for each of the 19 data sets used separately and, finally, the 19 contributions were added in quadrature to obtain the total contribution.

2. The variation of the data within the upper and lower limits of the NMC $F_{2}$ parameterization [49].

When calculating $g_{1}\left(x, Q^{2}\right)$ from the asymmetry data, see Section 3 , the NMC $F_{2}$ parameterization was used at its upper and lower limit to determine the changes in the polarized distributions compared to the 'central' curve. The extreme deviations from that curve were taken as the systematic uncertainties arising from the $F_{2}$ parameterization. 
3. The variation of the data within the uncertainty of the $R$ parameterization [46]. When calculating $g_{1}\left(x, Q^{2}\right)$ from the asymmetry data, see Section 3, the SLAC $R_{1990}$ parameterization was used at its uncertainty limits, see Ref. [46], to determine the changes in the spin-dependent distributions compared to the 'central' curve. The maximal deviations from that curve were taken as the systematic uncertainties arising from the $R$-parameterization.

Finally, the contributions from all three sources were added in quadrature to obtain the total contribution at each value of $x$, which is shown as hatched error bands in the figures below.

\subsection{Determination of theoretical systematic uncertainties}

The theoretical systematic uncertainties were estimated from the following sources:

1. The variation of the factorization and the renormalization scale by a factor of 2 .

2. An additional variation of $\Lambda_{\mathrm{QCD}}^{(4)}$. We varied $\Lambda_{\mathrm{QCD}}^{(4)}$ by $\pm 30 \mathrm{MeV}$, which corresponds to a variation of $\alpha_{s}\left(M_{Z}^{2}\right)$ by \pm 0.002 , being a typical error in individual precision measurements, in addition to the error of $\alpha_{S}\left(M_{Z}^{2}\right)$ being determined in the present fit.

3. The variation of $\eta_{u_{v}}$ and $\eta_{d_{v}}$ within the errors of the parameters $F$ and $D$, see Section 4, while keeping $g_{A} / g_{V}=F+D$ constant.

4. The variation of the parameterizations at the input scale $Q_{0}^{2}$.

Two cases are considered: first, the values of the parameter $\rho=-1.0$ for $\Delta u_{v}$ and $\Delta d_{v}$ are added in the fit. Second, the values of the parameter $\gamma=17.64$ for $\Delta u_{v}$ and 24.26 for $\Delta d_{v}$, respectively, for the same densities are changed compared to the values used for the central curve.

5. The variation of the standard input scale: from $Q_{0}^{2}=4.0 \mathrm{GeV}^{2}$ to $2.0 \mathrm{GeV}^{2}$ and $6.0 \mathrm{GeV}^{2}$.

For all items always the extreme deviations from the 'central' curve were taken as the contribution to the theoretical systematic uncertainty from that source. Finally, all different contributions were added in quadrature to get the total contribution at each value of $x$ which is shown as the second hatched error bands in the figures below.

\section{Results of the QCD analysis}

\subsection{The parton distributions}

In the NLO QCD fit we covered the polarized world deep-inelastic data with $Q^{2}>1 \mathrm{GeV}^{2}$, $W^{2}>3.24 \mathrm{GeV}^{2}$, with a value of $\chi^{2} / N D F=1.12$. We have chosen a value UP $=9.3$ corresponding to the $7+1$ parameter fit in the MINUIT-procedure. The parton distributions are parameterized at $Q_{0}^{2}=4 \mathrm{GeV}^{2}$. In Table 2 the values of the fit parameters are summarized. The covariance matrix of the fit is given in Table 3. Gaussian error propagation allows to derive the error bands due to the parton densities for the various polarized observables.

In Fig. 1 the polarized momentum distributions $x \Delta f_{i}(x)$ are presented at the scale $Q_{0}^{2}$. We compare with other analyses $[18,19,21,25]$. The $x \Delta u_{v}$ distribution is slightly lowered if compared to our previous analysis [23]. The most important change concerns the gluon distribution $x \Delta G$, which is lowered by about a factor of two relative to the results of Ref. [23]. Comparing to the results of other analyses $x \Delta u_{v}$ turns out to be lower in a wider range. In case of 
Table 2

Final parameter values and their statistical errors at the input scale $Q_{0}^{2}=4.0 \mathrm{GeV}^{2}$.

\begin{tabular}{|c|c|c|c|c|c|}
\hline \multirow[t]{4}{*}{$\Delta u_{v}$} & $\eta$ & 0.928 (fixed) & \multirow[t]{4}{*}{$\Delta \bar{q}_{s}$} & $\eta$ & $-0.066 \pm 0.013$ \\
\hline & $a$ & $0.239 \pm 0.027$ & & $a$ & $0.365 \pm 0.164$ \\
\hline & $b$ & $3.031 \pm 0.178$ & & $b$ & 8.080 (fixed) \\
\hline & $\gamma$ & 27.64 (fixed) & & $\gamma$ & 0.0 (fixed) \\
\hline \multirow[t]{4}{*}{$\Delta d_{v}$} & $\eta$ & -0.342 (fixed) & \multirow[t]{4}{*}{$\Delta G$} & $\eta$ & $0.462 \pm 0.430$ \\
\hline & $a$ & $0.128 \pm 0.068$ & & $a$ & $a_{\Delta \bar{q}_{s}}+1$ \\
\hline & $b$ & $4.055 \pm 0.879$ & & $b$ & 5.610 (fixed) \\
\hline & $\gamma$ & 44.26 (fixed) & & $\gamma$ & 0.0 (fixed) \\
\hline \multicolumn{3}{|c|}{$\Lambda_{\mathrm{QCD}}^{(4)}=243 \pm 62 \mathrm{MeV}$} & \multicolumn{3}{|c|}{$\chi^{2} / N D F=1537 / 1377=1.12$} \\
\hline
\end{tabular}

Table 3

The covariance matrix for the $7+1$ parameter NLO fit based on the world asymmetry data.

\begin{tabular}{|c|c|c|c|c|c|c|c|c|}
\hline & $\Lambda_{\mathrm{QCD}}^{(4)}$ & $a_{u_{v}}$ & $b_{u_{v}}$ & $a_{d_{v}}$ & $b_{d_{v}}$ & $\eta_{\text {sea }}$ & $a_{\text {sea }}$ & $\eta_{G}$ \\
\hline$\Lambda_{\mathrm{QCD}}^{(4)}$ & $3.84 E-3$ & & & & & & & \\
\hline$a_{u_{v}}$ & $-4.08 \mathrm{E}-4$ & $7.56 \mathrm{E}-4$ & & & & & & \\
\hline$b_{u_{v}}$ & $-1.14 \mathrm{E}-3$ & $4.30 \mathrm{E}-3$ & $3.18 \mathrm{E}-2$ & & & & & \\
\hline$a_{d_{v}}$ & $2.74 \mathrm{E}-3$ & $-9.39 \mathrm{E}-4$ & $-4.43 \mathrm{E}-3$ & $4.60 \mathrm{E}-3$ & & & & \\
\hline$b_{d_{v}}$ & $2.38 \mathrm{E}-2$ & $-8.33 \mathrm{E}-3$ & $-1.03 \mathrm{E}-2$ & $4.50 \mathrm{E}-2$ & $7.72 \mathrm{E}-1$ & & & \\
\hline$\eta_{\text {sea }}$ & $1.70 \mathrm{E}-3$ & $-6.84 \mathrm{E}-4$ & $-3.60 \mathrm{E}-3$ & $2.26 \mathrm{E}-3$ & $2.11 \mathrm{E}-2$ & $5.62 \mathrm{E}-3$ & & \\
\hline$a_{\text {sea }}$ & $-5.64 \mathrm{E}-3$ & $3.04 \mathrm{E}-3$ & $1.65 \mathrm{E}-2$ & $-8.25 \mathrm{E}-3$ & $-7.37 \mathrm{E}-2$ & $7.13 \mathrm{E}-4$ & $2.70 \mathrm{E}-2$ & \\
\hline$\eta_{G}$ & $-1.96 \mathrm{E}-2$ & $8.32 \mathrm{E}-3$ & $4.25 \mathrm{E}-2$ & $-2.16 \mathrm{E}-2$ & $-1.67 \mathrm{E}-1$ & $-2.09 \mathrm{E}-2$ & $4.17 \mathrm{E}-2$ & $1.85 \mathrm{E}-1$ \\
\hline
\end{tabular}

the $x \Delta d_{v}$-distribution all fits widely agree within the $1 \sigma$ error band. This also applies for the $x \Delta \bar{q}$-distribution. For the polarized gluon distribution the agreement of the different fits at larger values of $x$ agree within the $1 \sigma$ error, while below $x \sim 0.02$ the fits $[18,19,21]$ yield slightly higher values. We indicated the positivity constraints referring to the unpolarized PDFs [30], which are obeyed.

In Figs. 2 and 3 we analyze the systematic errors in more detail, cf. Sections 5.2 and 5.3. For the gluon distribution function $x \Delta G\left(x, Q_{0}^{2}\right)$, Fig. 2, the experimental systematic errors are lower than the statistical errors, but are still significant. The combined theoretical systematic effects at NLO amount to larger values than the experimental ones. About half of the error is due to the uncertainty in $\Lambda_{\mathrm{QCD}}$. Clearly, in future analyses based on NNLO QCD evolution this error will diminish. The effect of the experimental and theoretical systematic errors in case of the singlet distribution $x \Delta \Sigma\left(x, Q_{0}^{2}\right)$ is similar. The errors are smaller if compared to the gluon distribution. The singlet distribution at $Q_{0}^{2}=4 \mathrm{GeV}^{2}$ is negative (within the $1 \sigma$ errors) for $x<2 \cdot 10^{-2}$ and turns to positive values for $x>4 \cdot 10^{-2}$. All the fits $[18,19,21]$ lie inside the $1 \sigma$ error band. In the medium $x$-range the DSSV distribution [25] yields somewhat larger values.

In Fig. 4 we compare the fit results for the structure functions $g_{1}^{p}\left(x, Q^{2}\right), g_{1}^{d}\left(x, Q^{2}\right)$ and $g_{1}^{n}\left(x, Q^{2}\right)$ at $Q^{2}=5 \mathrm{GeV}^{2}$ with the data, cf. Table 1 , to illustrate the fit quality for the different targets as an example. Furthermore, also the results of the GRSV [19] and AAC [18] analyses are shown. Overall a good agreement is obtained.

A further illustration of the fit quality is presented in Fig. 5. Here the data for $g_{1}^{p}\left(x, Q^{2}\right)$ are compared to the fit including, the statistical errors. We also show the fit results of AAC, GRSV, and LSS $[18,19,21]$. Within the error bands the data agree well with the fit. In the lowest $x$-bins 

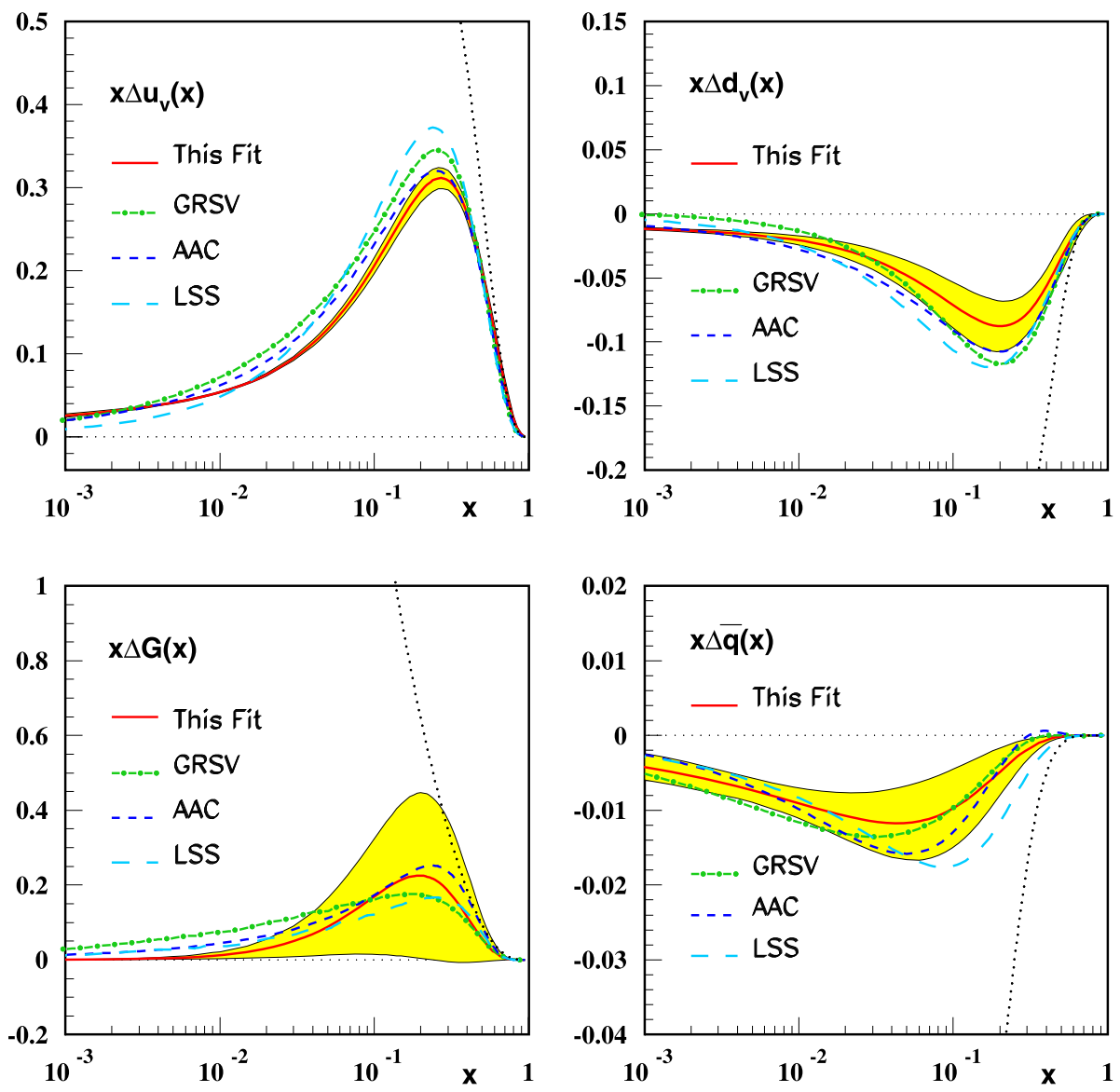

Fig. 1. NLO polarized parton distributions at the input scale $Q_{0}^{2}=4.0 \mathrm{GeV}^{2}$ (solid line) compared to results obtained by GRSV (dashed-dotted line) [19], DSSV (long dashed-dotted line) [25], AAC (dashed line) [18], and LSS (long dashed line) [21]. The shaded areas represent the fully correlated $1 \sigma$ error bands calculated by Gaussian error propagation.

the fluctuation is somewhat larger. In some cases the EMC-data [1] lay outside the $1 \sigma$ error range.

\section{2. $\Lambda_{\mathrm{QCD}}$ and $\alpha_{S}\left(M_{Z}^{2}\right)$}

The NLO QCD-analysis of the polarized world deep-inelastic data requires the fit of $\Lambda_{\mathrm{QCD}}$ along with the parameters of the non-perturbative parton distributions at the scale $Q_{0}^{2}$. As outlined in Table 3 the QCD-scale is correlated to all other parameters and in particular also to $\eta_{G}$. Due to this analyses in which $\alpha_{S}\left(M_{Z}^{2}\right)$ or $\Lambda_{\mathrm{QCD}}$ is imported from 3rd sources may suffer significant biases, i.e. a too large value of $\alpha_{s}\left(M_{Z}^{2}\right)\left(\Lambda_{\mathrm{QCD}}\right)$ leads to a too small gluon distribution, aside of other effects. Despite of various precision measurements of the strong coupling constant based on theoretical NNLO (and partially even higher) precision, a thorough agreement on the value of $\alpha_{S}\left(M_{Z}^{2}\right)$ has not yet been reached, cf. e.g. [65]. Due to this $\Lambda_{\mathrm{QCD}}$ is determined in this analysis. We refer to $\Lambda_{\mathrm{QCD}}^{\mathrm{NLO},\left(N_{f}=4\right)}$ as the NLO value for 4 active flavors and obtain 


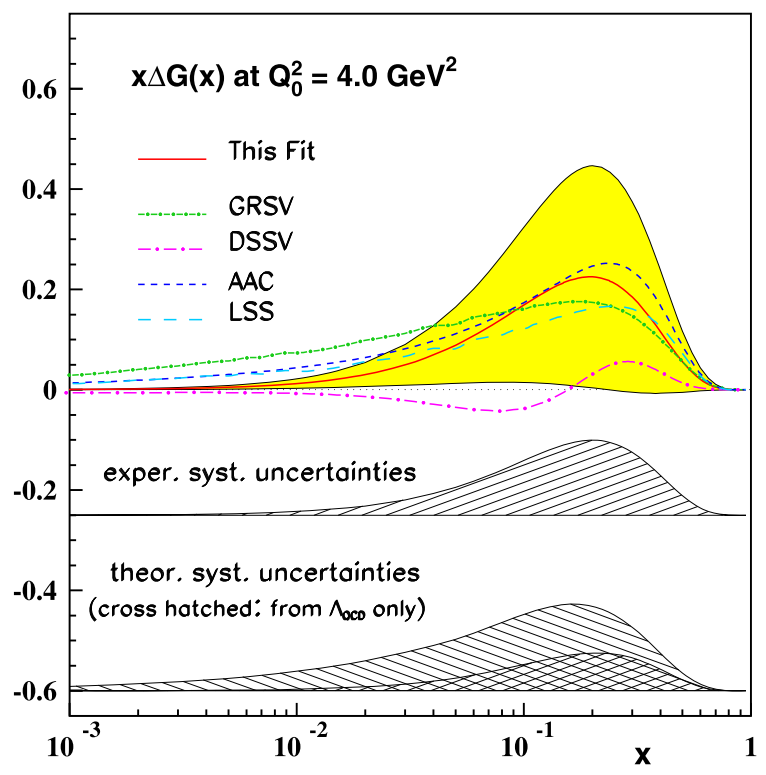

Fig. 2. The polarized parton density $x \Delta G(x)$ at $Q_{0}^{2}=4.0 \mathrm{GeV}^{2}$ as a function of $x$ (solid line). The shaded area is the fully correlated $1 \sigma$ statistical error band and the hatched areas are the systematic uncertainties. Results from GRSV (dasheddotted line) [19], DSSV (long dashed-dotted line) [25], AAC (dashed line) [18], and LSS (long dashed line) [21] are shown for comparison.

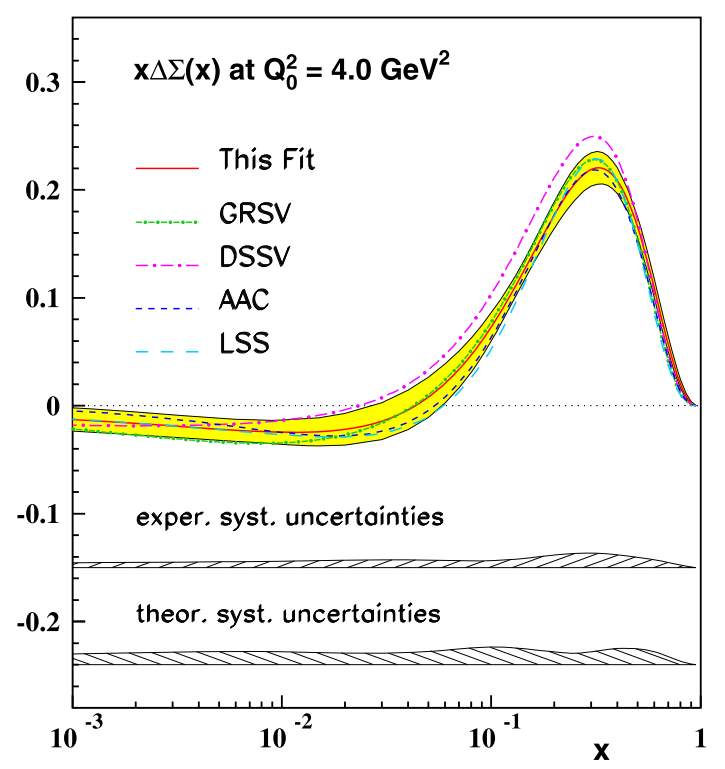

Fig. 3. The polarized parton density $x \Delta \Sigma(x)$ at $Q_{0}^{2}=4.0 \mathrm{GeV}^{2}$ as a function of $x$ (solid line). The shaded area is the fully correlated $1 \sigma$ statistical error band and the hatched areas are the systematic uncertainties. Results from GRSV (dasheddotted line) [19], DSSV (long dashed-dotted line) [25], AAC (dashed line) [18], and LSS (long dashed line) [21] are shown for comparison. 


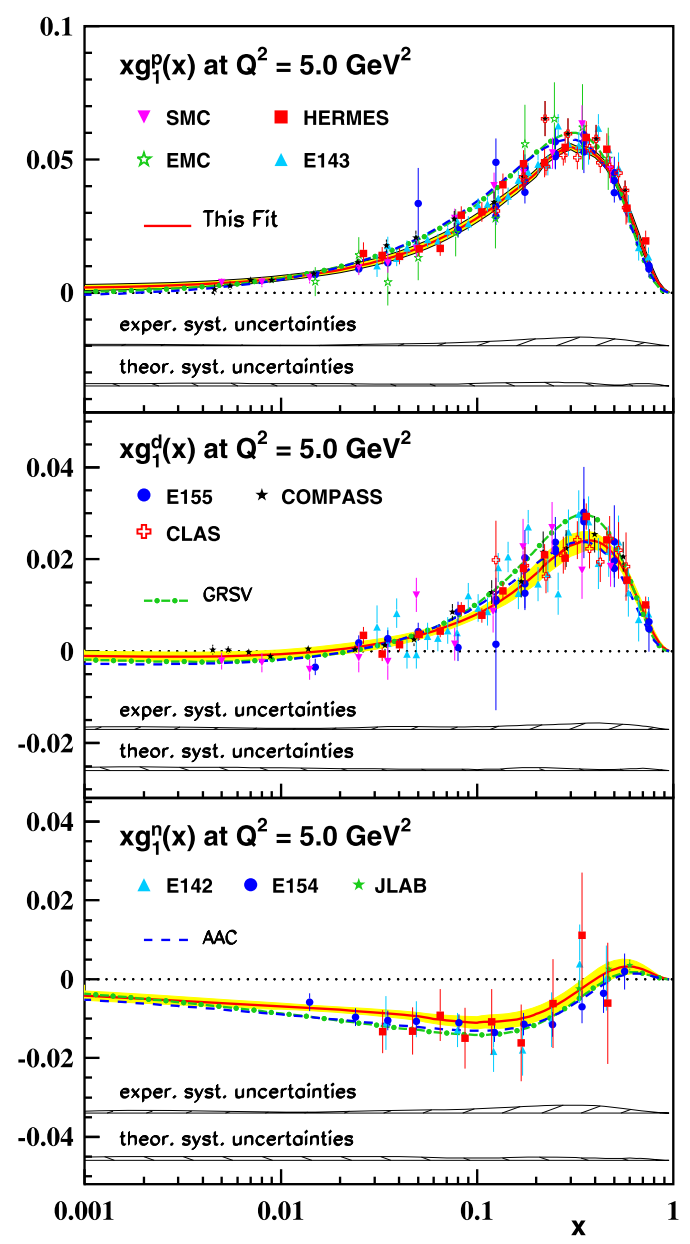

Fig. 4. The spin-dependent structure functions $x g_{1}^{p}(x), x g_{1}^{d}(x)$ and $x g_{1}^{n}(x)$ as a function of $x$. The experimental data are evolved to a common value of $Q^{2}=5 \mathrm{GeV}^{2}$. The error bars shown are the statistical and systematic ones added in quadrature. The experimental distributions are well described (solid curve) within the statistical (shaded areas) and systematic (hatched areas) error bands. The curves obtained by GRSV (dashed-dotted) [19] and AAC (dashed) [18] are shown for comparison.

$$
\Lambda_{\mathrm{QCD}}^{(4)}=243 \pm 62(\exp ) \mathrm{MeV} \text {. }
$$

In an earlier analysis [23] the values

$$
\begin{aligned}
& \Lambda_{\mathrm{QCD}}^{(4)}=235 \pm 53(\exp ) \mathrm{MeV} \quad \mathrm{ISET}=3, \\
& \Lambda_{\mathrm{QCD}}^{(4)}=240 \pm 60(\exp ) \mathrm{MeV} \quad \mathrm{ISET}=4
\end{aligned}
$$

were found, for comparison, slightly depending on some assumptions in the fit. The variation of the factorization and renormalization scales $\mu_{f, r}^{2}$ by a factor of $1 / 2$ and 2 , respectively, yields

$$
\Lambda_{\mathrm{QCD}}^{(4)}=243 \pm 62(\exp )_{+21}^{-37}(\mathrm{FS})_{-87}^{+46}(\mathrm{RS}) \mathrm{MeV} .
$$




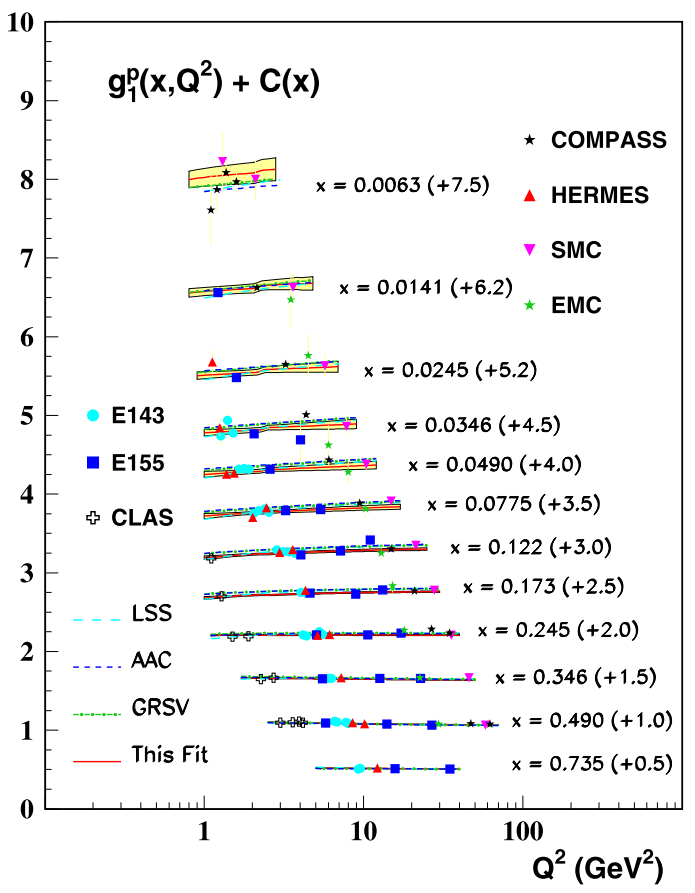

Fig. 5. The spin-dependent structure function $x g_{1}^{p}\left(x, Q^{2}\right)$ as a function of $x$ and $Q^{2}$. The experimental data are compared to the fit result (solid curve) with the statistical error bands (shaded areas). The curves obtained by GRSV (dasheddotted) [19], AAC (short dashed) [18] and LSS (long dashed) [21] are shown for comparison.

Here we excluded values $\mu_{f, r}^{2}<1 \mathrm{GeV}^{2}$, unlike in Ref. [23], since at scales lower than $1 \mathrm{GeV}^{2}$ the perturbative description cannot be considered reliable anymore.

Correspondingly, for $\alpha_{s}\left(M_{Z}^{2}\right)$ one obtains

$$
\alpha_{S}\left(M_{Z}^{2}\right)=0.1132_{-0.0051}^{+0.0043}(\exp )_{+0.0015}^{-0.0029}(\mathrm{FS})_{-0.0075}^{+0.0032}(\mathrm{RS}),
$$

with combined errors of

$$
\alpha_{s}\left(M_{Z}^{2}\right)=0.1132_{-0.0095}^{+0.0056} \text {. }
$$

Due to the NLO analysis the factorization and renormalization scale uncertainties are still dominant. The values are well compatible with recent determinations of the strong coupling constant at NNLO and $\mathrm{N}^{3} \mathrm{LO}$ from deep-inelastic data:

$$
\begin{array}{ll}
\alpha_{s}\left(M_{Z}^{2}\right)=0.1134_{-0.0021}^{+0.0019} \quad \text { NNLO [28], } \\
\alpha_{s}\left(M_{Z}^{2}\right)=0.1141_{-0.0022}^{+0.0020} \quad \mathrm{~N}^{3} \text { LO [28], } \\
\alpha_{s}\left(M_{Z}^{2}\right)=0.1135 \pm 0.0014 \quad \text { NNLO, FFS [31], } \\
\alpha_{s}\left(M_{Z}^{2}\right)=0.1129 \pm 0.0014 \quad \text { NNLO, BSMN [31], } \\
\alpha_{s}\left(M_{Z}^{2}\right)=0.1124 \pm 0.0020 \quad \text { NNLO, dyn. approach [29], } \\
\alpha_{s}\left(M_{Z}^{2}\right)=0.1158 \pm 0.0035 \quad \text { NNLO, stand. approach [29], } \\
\alpha_{s}\left(M_{Z}^{2}\right)=0.1171 \pm 0.0014 & \text { NNLO [30]. }
\end{array}
$$




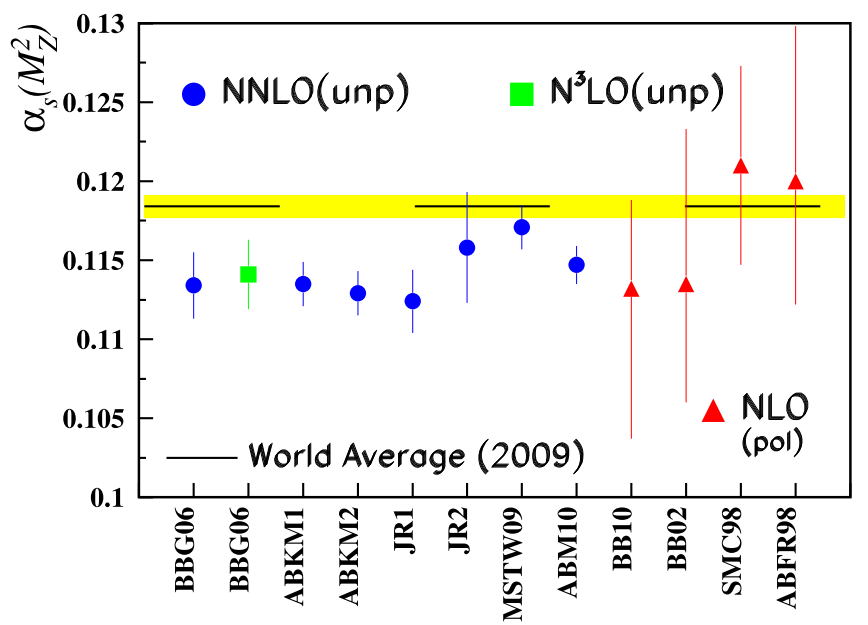

Fig. 6. The strong coupling constant $\alpha_{S}\left(M_{Z}^{2}\right)$ from different DIS measurements, Eqs. (49)-(56) and Refs. [17,22,25]. The yellow band describes the weighted average of a wide range of $\alpha_{S}\left(M_{Z}\right)$ measurements [65].

More recent unpolarized NNLO fits, including the combined HERA data [66], yield

$$
\begin{array}{ll}
\alpha_{s}\left(M_{Z}^{2}\right)=0.1147 \pm 0.0012 & \text { NNLO [67] } \\
\alpha_{s}\left(M_{Z}^{2}\right)=0.1145 \pm 0.0042 & \text { NNLO (preliminary) [68]. }
\end{array}
$$

The central value of the present fit (48) does well compare with the above values. They are located below the present weighted average of $\alpha_{s}\left(M_{Z}^{2}\right)$ measurements [65] of

$$
\alpha_{S}\left(M_{Z}^{2}\right)=0.1184 \pm 0.0007
$$

The error given in (58) does not include any yet unknown relative systematics between the different classes of the same type of measurement.

We would like to mention that recent determinations of $\alpha_{s}\left(M_{Z}^{2}\right)$ using event shape moments for high energy $e^{+} e^{-}$annihilation data from PETRA and LEP including power corrections the following values were obtained:

$$
\begin{aligned}
& \alpha_{S}\left(M_{Z}^{2}\right)=0.1135 \pm 0.0002(\exp ) \pm 0.005\left(\Omega_{1}\right) \pm 0.0009 \text { (pert) } \quad \text { NNLO [69] } \\
& \alpha_{S}\left(M_{Z}^{2}\right)=0.1153 \pm 0.0017(\exp ) \pm 0.0023(\text { th }) \quad \text { NNLO [70]. }
\end{aligned}
$$

Also these measurements of $\alpha_{s}\left(M_{Z}^{2}\right)$ yield low values. They show that the results obtained analyzing deep-inelastic data do not form a special case. The systematics of the different extractions of $\alpha_{S}\left(M_{Z}^{2}\right)$ has to be understood in more detail in the future.

Fit results from previous polarized analyses like [15,17,26] were summarized in [23]. In Fig. 6 we compare recent determinations at NNLO and $\mathrm{N}^{3} \mathrm{LO}$ for unpolarized and at NLO for polarized deep-inelastic scattering.

\subsection{Moments of polarized parton distributions}

We calculate the lowest moments of the polarized parton densities 
Table 4

Moments of the NLO parton densities and their combinations for the present analysis at $Q^{2}=4 \mathrm{GeV}^{2}$. The value of the respective moment integrating only outside the $x$-range in which currently deep-inelastic scattering data are measured, $0.005<x<0.75$, are given for comparison (lowerlupper part). The errors are the $1 \sigma$ correlated errors.

\begin{tabular}{|c|c|c|c|c|}
\hline \multirow[t]{2}{*}{ Distribution } & \multicolumn{3}{|c|}{ Fit results } & \multirow[t]{2}{*}{ [23], set 3} \\
\hline & $n$ & Value & Value out of range & \\
\hline \multirow[t]{4}{*}{$\Delta u_{v}$} & 0 & $0.928 \pm 0.000$ & $0.158 \mid 3.3 \mathrm{E}-3$ & $0.926 \pm 0.071$ \\
\hline & 1 & $0.153 \pm 0.004$ & $1.6 \mathrm{E}-4 \mid 2.7 \mathrm{E}-3$ & $0.163 \pm 0.014$ \\
\hline & 2 & $0.052 \pm 0.002$ & $0 \mid 2.1 \mathrm{E}-3$ & $0.055 \pm 0.006$ \\
\hline & 3 & $0.023 \pm 0.001$ & $011.7 \mathrm{E}-3$ & $0.024 \pm 0.003$ \\
\hline \multirow[t]{4}{*}{$\Delta d_{v}$} & 0 & $-0.342 \pm 0.000$ & $-0.110 \mid-2.1 \mathrm{E}-4$ & $-0.341 \pm 0.123$ \\
\hline & 1 & $-0.037 \pm 0.007$ & $-7.0 \mathrm{E}-5 \mathrm{I}-1.7 \mathrm{E}-4$ & $-0.047 \pm 0.021$ \\
\hline & 2 & $-0.010 \pm 0.002$ & $0 \mid-1.3 \mathrm{E}-4$ & $-0.015 \pm 0.009$ \\
\hline & 3 & $-0.004 \pm 0.001$ & $0 \mid-1.1 \mathrm{E}-4$ & $-0.006 \pm 0.005$ \\
\hline \multirow[t]{4}{*}{$\Delta u-\Delta d$} & 0 & $1.270 \pm 0.000$ & $0.267 \mid 3.5 \mathrm{E}-3$ & $1.267 \pm 0.142$ \\
\hline & 1 & $0.190 \pm 0.008$ & $2.3 \mathrm{E}-4 \mid 2.8 \mathrm{E}-3$ & $0.210 \pm 0.025$ \\
\hline & 2 & $0.063 \pm 0.004$ & $0 \mid 2.3 \mathrm{E}-3$ & $0.070 \pm 0.011$ \\
\hline & 3 & $0.027 \pm 0.002$ & $0 \mid 1.8 \mathrm{E}-3$ & $0.030 \pm 0.006$ \\
\hline \multirow[t]{4}{*}{$\Delta u$} & 0 & $0.866 \pm 2 \mathrm{E}-5$ & $0.136 \mid 3.3 \mathrm{E}-3$ & $0.851 \pm 0.075$ \\
\hline & 1 & $0.151 \pm 0.004$ & $1.3 \mathrm{E}-4 \mid 2.7 \mathrm{E}-3$ & $0.160 \pm 0.014$ \\
\hline & 2 & $0.052 \pm 0.002$ & $0 \mid 2.1 \mathrm{E}-3$ & $0.055 \pm 0.006$ \\
\hline & 3 & $0.023 \pm 0.001$ & $0 \mid 1.7 \mathrm{E}-3$ & $0.024 \pm 0.003$ \\
\hline \multirow[t]{4}{*}{$\Delta d$} & 0 & $-0.404 \pm 3 E-5$ & $-0.132 \mathrm{I}-2.1 \mathrm{E}-4$ & $-0.415 \pm 0.124$ \\
\hline & 1 & $-0.039 \pm 0.007$ & $-1.0 \mathrm{E}-4 \mathrm{I}-1.7 \mathrm{E}-4$ & $-0.050 \pm 0.022$ \\
\hline & 2 & $-0.011 \pm 0.002$ & $-0 \mid-1.3 \mathrm{E}-4$ & $-0.015 \pm 0.009$ \\
\hline & 3 & $-0.004 \pm 0.001$ & $0 \mid-1.1 \mathrm{E}-4$ & $-0.006 \pm 0.005$ \\
\hline \multirow[t]{4}{*}{$\Delta \bar{q}$} & 0 & $-0.066 \pm 0.013$ & -0.0210 & $-0.074 \pm 0.017$ \\
\hline & 1 & $-2.5 \mathrm{E}-3 \pm 1.2 \mathrm{E}-3$ & $-3.0 \mathrm{E}-510$ & $-0.003 \pm 0.001$ \\
\hline & 2 & $-3.3 \mathrm{E}-4 \pm 2.0 \mathrm{E}-4$ & $0 \mid 0$ & $-4.0 \mathrm{E}-4 \pm 1.0 \mathrm{E}-4$ \\
\hline & 3 & $-7.0 \mathrm{E}-5 \pm 4.0 \mathrm{E}-5$ & 010 & $-8.0 \mathrm{E}-5 \pm 2.0 \mathrm{E}-5$ \\
\hline \multirow[t]{4}{*}{$\Delta G$} & 0 & $0.462 \pm 0.430$ & $0.004 \mid 1.0 \mathrm{E}-4$ & $1.062 \pm 0.549$ \\
\hline & 1 & $0.079 \pm 0.079$ & $1.0 \mathrm{E}-5 \mid 8.0 \mathrm{E}-5$ & $0.184 \pm 0.103$ \\
\hline & 2 & $0.021 \pm 0.021$ & $0 \mid 6.3 \mathrm{E}-5$ & $0.050 \pm 0.028$ \\
\hline & 3 & $0.007 \pm 0.007$ & $0 \mid 4.9 \mathrm{E}-5$ & $0.017 \pm 0.010$ \\
\hline
\end{tabular}

$$
\langle f(x)\rangle_{n}=\int_{0}^{1} d x x^{n} \Delta f(x),
$$

where $\Delta f(x)$ denote the different polarized (number) density distributions. The moments $n=$ $0, \ldots, 3$ are given in Table 4 . The behaviour of these distributions outside the kinematic range in which the fit is performed bear uncertainties, which are difficult to predict for these nonperturbative quantities. ${ }^{6}$ Instead of presenting necessarily uncertain models for this range, we

\footnotetext{
6 We remind the failure in predicting the lower $x$ behaviour of the structure function $F_{2}\left(x, Q^{2}\right)$ prior the HERA measurements until 1992, which assumed a slightly falling or constant behaviour below $x \simeq 10^{-2}$, whereas a strong rise was measured at HERA.
} 
compute the respective part of the moments for values $x<0.005$ and $x>0.75$ extrapolating the present distributions to the range $x \in[0,1]$.

The zeroth moments of the polarized quark and gluon distributions as well as the contributions due to the quark and gluon angular momenta, $L_{q}$ and $L_{g}$, constitute the nucleon spin

$$
\frac{1}{2}=\frac{1}{2}\langle\Delta \Sigma(x)\rangle_{0}+\langle\Delta G(x)\rangle_{0}+L_{q}+L_{g} .
$$

We obtain

$$
\begin{aligned}
& \left\langle\Delta \Sigma\left(x, Q_{0}^{2}\right)\right\rangle_{0}=0.193 \pm 0.075, \\
& \left\langle\Delta G\left(x, Q_{0}^{2}\right)\right\rangle_{0}=0.462 \pm 0.430,
\end{aligned}
$$

at $Q_{0}^{2}=4 \mathrm{GeV}^{2}$. For Eq. (62) this yields

$$
\frac{1}{2}=(0.555 \pm 0.436)+L_{q}+L_{g} .
$$

The error of $\Delta G$ is clearly dominant.

The results given in Table 4 can be compared to ab initio calculations of these moments in Lattice Gauge Theory. There we also compare with the values obtained in our previous analysis [23]. In the present analysis the first moments $(n=0)$ of the polarized valence quark distributions are determined by the values of $F$ and $D$ and are fixed in the fit. Comparing to the results of [23], ISET $=3^{7}$ slightly larger values are obtained for the moments of $\Delta u_{v}\left(x, Q^{2}\right)$ and slightly lower values for $\Delta d_{v}\left(x, Q^{2}\right)$ and $\Delta \bar{q}\left(x, Q^{2}\right)$. A very significant change is obtained for the moments of the polarized gluon density, where the moments reduced by a factor of about two comparing to [23]. Although being positive, the latter moments are now compatible with zero in the $1 \sigma$ errors.

First lattice results for the moments $n=0,1,2$ of the polarized quark distributions were given about ten years ago. Meanwhile many systematic effects in the simulation have been improved further. Still there are differences in the different simulations. Rather aiming on a detailed comparison with the values in Table 4 we give a brief summary of the current status. A recent survey has been presented in [71]. Lattice results on $\langle\Delta u-\Delta d\rangle_{0}$ for $m_{\pi}^{2}=0.029 \ldots 0.48 \mathrm{GeV}^{2}$ were given in [72-75] by the BGR, RBC, LHPC, ETMC, QCDSF Collaborations using dynamical quarks. Most of the values are yet below the experimental value. The QCDSF Collaboration [75] performed simulations at $m_{\pi}=170 \mathrm{MeV}$ and obtained

$$
\begin{aligned}
& \langle\Delta u(x)-\Delta d(x)\rangle_{0}=1.17 \pm 0.05 \\
& \frac{\langle\Delta u(x)-\Delta d(x)\rangle_{0}}{\langle\Delta u(x)+\Delta d(x)\rangle_{0}}=0.47 \pm 0.02
\end{aligned}
$$

For the first moment the following values were determined

$$
\begin{aligned}
\langle\Delta u-\Delta d\rangle_{1} & =0.271 \pm 0.040, & & m_{\pi}=493 \mathrm{MeV} \\
& =0.252 \pm 0.020, & & m_{\pi}=352 \mathrm{MeV}
\end{aligned}
$$

which are larger than the value

$$
\langle\Delta u-\Delta d\rangle_{1}=0.190 \pm 0.008
$$

determined in the present analysis.

\footnotetext{
7 The NLO results for ISET $=4$ are quite similar.
} 
Results on the second moment were given in [74]

$$
\langle\Delta u-\Delta d\rangle_{2}=0.083 \pm 0.012, \quad m_{\pi}=352 \mathrm{MeV},
$$

to be compared to

$$
\langle\Delta u-\Delta d\rangle_{2}=0.063 \pm 0.004
$$

obtained in this analysis.

Results of older lattice simulations [76] were discussed in [23] previously. The values given above for the 1st and 2nd moment are based on theoretically much improved simulations, if compared to early investigations [76]. Yet, the moments obtained yield similar values. Comparing the lattice results with the results obtained in QCD-fits to the polarized deep-inelastic world data one observes a similar trend of values but not yet agreement.

\section{Higher twist}

So far we have applied the twist-2 approximation at NLO to describe the spin-dependent structure function $g_{1}$. As the data may contain contributions from higher twist (HT) it is of interest to look for possible effects of such contributions. A thorough description of higher twist anomalous dimensions and Wilson coefficients to NLO is still missing, even for the twist-4 contributions. Therefore we will perform a purely phenomenological analysis. Similar to the approach of Ref. [77] for the structure function $F_{2}$, where a higher twist term is parameterized by the ansatz

$$
h_{H T}^{i}\left(x, Q^{2}\right)=\frac{C_{i}(x)}{Q^{2}},
$$

and used multiplicative to the leading twist (LT) contribution, $g_{1}\left(x, Q^{2}\right)$ is described by

$$
g_{1}^{i}\left(x, Q^{2}\right)=g_{1, L T}^{i}\left(x, Q^{2}\right)\left[1+h_{H T, m}^{i}\left(x, Q^{2}\right)\right],
$$

where $i=p, d, n$. Likewise one may describe an additive higher twist term

$$
g_{1}^{i}\left(x, Q^{2}\right)=g_{1, L T}^{i}\left(x, Q^{2}\right)+h_{H T, a}^{i}\left(x, Q^{2}\right) .
$$

This approach has to be handled with great care, since the coefficients $C_{i}(x)$ are actually also $Q^{2}$ dependent. They consist of a combination of various terms which exhibit different scaling violations. The relation to $\Lambda_{\mathrm{QCD}}$ is completely masked here. Moreover, higher twist contributions should have a flavor-dependence and are not expected to be the same in case of polarized and unpolarized deep-inelastic scattering.

The kinematic $x$-range being covered by experiment is divided into 5 bins and the coefficient $C_{i}(x)$ has to be determined in each bin and for each target. The resulting coefficients for the proton and the deuteron target, $C_{p}(x)$ and $C_{d}(x)$, are summarized in Table 5 both for the multiplicative and additive cases. Due to the enlarged number of parameters being fitted the value of UP $=11.5$ is used. In the additive and multiplicative cases we observe quite comparable patterns. We prefer the additive case, since the twist- 2 scaling violations of $g_{1}\left(x, Q^{2}\right)$ do not influence $C_{p, d, n}(x)$. Here, in most of the bins the result is $1 \sigma$ compatible with zero, except of one case for $C_{p}(x) . C_{d, n}(x)$ are all $1 \sigma$ compatible with zero. The behaviour in case of the deuteron and neutron is rather flat, while for the proton a slight structure, yet with large errors, is indicated, cf. Fig. 7. In the fit determining also the higher twist parameters the value $\chi^{2} / N D F$ for the CLAS data amounts to 1.12. The present data are not yet precise enough to undoubtedly reveal higher 
Table 5

The higher twist coefficients $C_{p}(x)$ and $C_{d}(x)$ as function of $x$ considering both a multiplicative and additive phenomenological ansatz.

\begin{tabular}{|c|c|c|c|c|}
\hline \multirow[t]{2}{*}{$\langle x\rangle$} & $C_{p}\left[\mathrm{GeV}^{2}\right]$ & $C_{d}\left[\mathrm{GeV}^{2}\right]$ & $C_{p}\left[\mathrm{GeV}^{2}\right]$ & $C_{d}\left[\mathrm{GeV}^{2}\right]$ \\
\hline & \multicolumn{2}{|l|}{ Multiplicative } & \multicolumn{2}{|l|}{ Additive } \\
\hline 0.060 & $-0.084 \pm 0.245$ & $0.007 \pm 0.287$ & $0.011 \pm 0.076$ & $0.019 \pm 0.059$ \\
\hline 0.150 & $-0.229 \pm 0.156$ & $0.169 \pm 0.431$ & $-0.038 \pm 0.037$ & $0.022 \pm 0.042$ \\
\hline 0.275 & $-0.224 \pm 0.099$ & $-0.226 \pm 0.270$ & $-0.045 \pm 0.022$ & $-0.007 \pm 0.025$ \\
\hline 0.425 & $-0.083 \pm 0.140$ & $-0.013 \pm 0.384$ & $-0.017 \pm 0.021$ & $0.005 \pm 0.027$ \\
\hline 0.625 & $0.290 \pm 0.417$ & $0.061 \pm 1.106$ & $0.011 \pm 0.033$ & $0.011 \pm 0.031$ \\
\hline
\end{tabular}

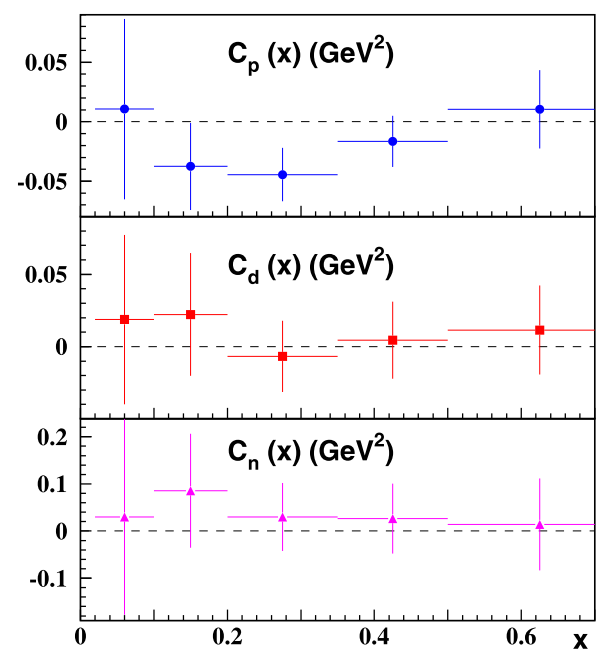

Fig. 7. The additive higher twist coefficients $C_{p}(x), C_{d}(x)$ and $C_{n}(x)$ as a function of $x$.

twist contributions in the range $Q^{2}>1 \mathrm{GeV}^{2}$ and a NLO QCD analysis can be carried out in the leading twist approximation. We do not confirm the results of Ref. [22]. Unlike the case for the large $x$ valence quark region, in which dynamical higher twist terms are extracted consistently in the unpolarized case, cf. Refs. [28,78,79], the situation is more involved for the lower $x$-region. The dynamics is clearly different in both these domains due to the contributing parton species. As has been shown in [80], different power corrections cancel each other in the small $x$ region.

\section{Conclusions}

A QCD analysis of the polarized deep-inelastic world data has been performed at NLO, including the effects of charm production to first order. We derived a parameterization for the polarized parton distributions and $\Lambda_{\mathrm{QCD}}^{N_{f}=4}$ with the error correlations between the fitted parameters applying the $\chi^{2}$-method. Detailed comparisons have been performed with recent parameterizations $[18,19,21,25]$. The present data are not accurate enough to determine all the shape parameters at a sufficient accuracy. Due to this some of the parameters have to be fixed after an initial phase of the analysis to a model-value. If compared to our previous analysis [23] the more recent data lead to a smaller gluon distribution, which is for a wide region of $x$ compatible with 
zero within the $1 \sigma$ error. We determined both the experimental and theoretical systematic effects. Both the central values of the parton densities and their $1 \sigma$ error are made available in form of a numerical parameterization in the range $x \in\left[10^{-9}, 1\right], Q^{2} \in\left[1,10^{6}\right] \mathrm{GeV}^{2}$. These distributions can be used for polarized hard-scattering processes at hadron-nucleon and lepton-nucleon colliders for various observables, including error propagation w.r.t. the accuracy of the parton densities. The implementation in terms of grid-interpolation is well suited also for Monte Carlo simulations.

The QCD-scale was determined by $\Lambda_{\mathrm{QCD}}^{N_{f}=4}=243 \pm 62(\exp )_{-90}^{+59}($ th) $\mathrm{MeV}$, corresponding to $\alpha_{s}^{\mathrm{NLO}}\left(M_{Z}^{2}\right)=0.1132_{-0.0095}^{+0.0056}$. The central value is well compatible with other measurements, cf. [28,29,31,67-70]. The errors are still rather large, also because of the scale variation uncertainties at NLO. Nonetheless the correlated determination of $\alpha_{S}\left(M_{Z}^{2}\right)$ with the parton densities is of importance to avoid biases in particular w.r.t. to the size of the gluon distribution function.

We also determined potential higher twist contributions, which were found to be compatible with zero in the whole kinematic range within the present errors for $C_{n, d}(x)$ and also for $C_{p}(x)$, except for one of the bins. Based on the results of the present analysis we computed the lowest moments of the individual twist-2 parton densities. For the lowest moment $(1 / 2)\langle\Delta \Sigma(x)\rangle_{0}+$ $\langle\Delta G(x)\rangle_{0}$ we obtain $0.555 \pm 0.436$ at $Q_{0}^{2}=4 \mathrm{GeV}^{2}$, which is well compatible with the nucleon spin $1 / 2$ even without angular momentum contributions. However, the error is dominated by that of the polarized gluon distribution. The moments derived may be compared to upcoming lattice simulations based on dynamical quarks of the corresponding operator matrix elements. The present results are not yet in agreement, although the tendency of values is visible. Runs at even smaller values of $m_{\pi}$ seem to be necessary.

\section{Acknowledgements}

We would like to thank S. Alekhin, E. Aschenauer, L. De Nardo, M. Göckeler, K. Jansen, W.D. Nowak, D. Renner, and G. Schierholz for discussions. This work was supported in part by DFG Sonderforschungsbereich Transregio 9, Computergestützte Theoretische Teilchenphysik and the European Commission MRTN HEPTOOLS under Contract No. MRTN-CT-2006-035505.

\section{Appendix A. The FORTRAN-code for the parton densities and their errors}

A fast FORTRAN program is available to represent the polarized parton densities $x \Delta u_{v}(x$, $\left.Q^{2}\right), x \Delta d_{v}\left(x, Q^{2}\right), x \Delta G\left(x, Q^{2}\right)$, and $x \Delta \bar{q}\left(x, Q^{2}\right)$, as well as the polarized structure functions $x g_{1}^{p}\left(x, Q^{2}\right)$ and $x g_{1}^{n}\left(x, Q^{2}\right)$ at NLO in the $\overline{\mathrm{MS}}$-scheme together with the parameterizations of their $1 \sigma$ errors. The following ranges in $x$ and $Q^{2}$ are covered:

$$
10^{-9}<x<1, \quad 1 \mathrm{GeV}^{2}<Q^{2}<10^{6} \mathrm{GeV}^{2} \text {. }
$$

The polarized distributions are the result of a fit to the world data on spin asymmetries, i.e. $A_{1}^{p, n, d}$ or $g_{1} / F_{1}^{p, n, d}$, as described above. The SUBROUTINE POLPDF returns the values of the polarized distributions, always multiplied by $x$, at a given point in $x$ and $Q^{2}$ by interpolating the data on specified grids. The interpolation in $x$ is done by cubic splines and in $Q^{2}$ by a linear interpolation in $\log \left(Q^{2}\right) .^{8}$

\footnotetext{
8 We thank S. Kumano and M. Miyama of the AAC Collaboration for allowing us to use their interpolation routines.
} 
The parton distributions are evaluated by

SUBROUTINE POLPDF(ISET, X, Q2, UV, DUV, DV, DDV, GL, DGL, QB, DQB, G1P,

DG1P, G1N, DG1N),

with $\operatorname{ISET}=1$. All non-integer variables are of the type REAL $* 8$. The calling routine has to contain the COMMON/INTINI/IINI. Before the first call to SUBROUTINE POLPDF the initialization is set by IINI $=0$.

The parameters $\mathrm{X}, \mathrm{Q}^{2}\left[\mathrm{GeV}^{2}\right]$ are $x$ and $Q^{2}$. The momentum densities of the polarized up and down valence quarks, gluons and the sea quarks are UV, DV, GL, QB, with $\mathrm{QB}=x \Delta u_{s}=x \Delta d_{s}=x \Delta \bar{u}=x \Delta \bar{d}=x \Delta s=x \Delta \bar{s}$. Correspondingly, DUV is the $1 \sigma$ error of UV, etc. and G1P and G1N are the values of the electromagnetic structure functions $x g_{1}^{p}$ and $x g_{1}^{n}$.

The programme example.f reads the data-grid qcd_nlo_907_0.grid and is compiled using gfortran at a LINUX-system. The test-code produces the test-output for the structurefunctions $\mathrm{xg} 1 \mathrm{p}, \mathrm{xg} 1 \mathrm{n}, \mathrm{xg} 1 \mathrm{~d}$ and their $1 \sigma$ errors $\mathrm{dxg} 1 \mathrm{p}, \mathrm{dxg} 1 \mathrm{n}, \mathrm{dxg} 1 \mathrm{~d}$ :

$\begin{array}{llllllll}\text { * } \mathrm{x}, \mathrm{q} 2, \mathrm{~g} 1 \mathrm{p}, \mathrm{dg} 1 \mathrm{p}, \mathrm{g} 1 \mathrm{n}, \mathrm{dg} 1 \mathrm{n}, \mathrm{g} 1 \mathrm{~d}, \mathrm{dg} 1 \mathrm{~d} \\ 0.100000 & 4.000000 & 0.027274 & 0.001453 & -0.011366 & 0.001468 & 0.007358 & 0.000955 \\ 0.200000 & 4.000000 & 0.043553 & 0.001347 & -0.007264 & 0.001895 & 0.016784 & 0.001075 \\ 0.300000 & 4.000000 & 0.052548 & 0.001146 & -0.004617 & 0.001947 & 0.022168 & 0.001045 \\ 0.400000 & 4.000000 & 0.054270 & 0.001137 & 0.000329 & 0.002212 & 0.025252 & 0.001150 \\ 0.500000 & 4.000000 & 0.046195 & 0.001369 & 0.003331 & 0.002272 & 0.022906 & 0.001227 \\ 0.600000 & 4.000000 & 0.034999 & 0.001821 & 0.003411 & 0.001920 & 0.017764 & 0.001224 \\ 0.700000 & 4.000000 & 0.022119 & 0.001858 & 0.002789 & 0.001254 & 0.011520 & 0.001037 \\ 0.800000 & 4.000000 & 0.011919 & 0.001254 & 0.001508 & 0.000557 & 0.006210 & 0.000634 \\ 0.900000 & 4.000000 & 0.006516 & 0.000383 & 0.000476 & 0.000115 & 0.003234 & 0.000185 \\ 0.950000 & 4.000000 & 0.005713 & 0.000087 & 0.000252 & 0.000023 & 0.002759 & 0.000042\end{array}$

The program can be received on request via e-mail to Johannes. Bluemlein@desy. de or Helmut. Boettcher@desy . de or from http://www-zeuthen.desy.de/ blumlein.

\section{References}

[1] J. Ashman, et al., European Muon Collaboration, Phys. Lett. B 206 (1988) 364; J. Ashman, et al., European Muon Collaboration, Nucl. Phys. B 328 (1989) 1.

[2] P.L. Anthony, et al., E142 Collaboration, Phys. Rev. D 54 (1996) 6620, arXiv:hep-ex/9610007.

[3] K. Ackerstaff, et al., HERMES Collaboration, Phys. Lett. B 404 (1997) 383, arXiv:hep-ex/9703005.

[4] K. Abe, et al., E154 Collaboration, Phys. Rev. Lett. 79 (1997) 26, arXiv:hep-ex/9705012.

[5] B. Adeva, et al., Spin Muon Collaboration, Phys. Rev. D 58 (1998) 112001.

[6] K. Abe, et al., E143 Collaboration, Phys. Rev. D 58 (1998) 112003, arXiv:hep-ph/9802357.

[7] A. Airapetian, et al., HERMES Collaboration, Phys. Rev. D 75 (2007) 012007, arXiv:hep-ex/0609039.

[8] P.L. Anthony, et al., E155 Collaboration, Phys. Lett. B 463 (1999) 339, arXiv:hep-ex/9904002.

[9] P.L. Anthony, et al., E155 Collaboration, Phys. Lett. B 493 (2000) 19, arXiv:hep-ph/0007248.

[10] X. Zheng, et al., JLAB Hall A Collaboration, Phys. Rev. C 70 (2004) 065207.

[11] V.Y. Alexakhin, et al., COMPASS Collaboration, Phys. Lett. B 647 (2007) 8, arXiv:hep-ex/0609038.

[12] K.V. Dharmawardane, et al., CLAS Collaboration, Phys. Lett. B 641 (2006) 11, arXiv:nucl-ex/0605028.

[13] CLAS Collaboration, private communication.

[14] M.G. Alekseev, et al., COMPASS Collaboration, arXiv:1001.4654 [hep-ex].

[15] K. Abe, et al., E154 Collaboration, Phys. Lett. B 405 (1997) 180, arXiv:hep-ph/9705344.

[16] J. Blümlein, A. Tkabladze, Nucl. Phys. B 553 (1999) 427, arXiv:hep-ph/9812478.

[17] G. Altarelli, R.D. Ball, S. Forte, G. Ridolfi, Acta Phys. Polon. B 29 (1998) 1145, arXiv:hep-ph/9803237; S. Forte, M.L. Mangano, G. Ridolfi, Nucl. Phys. B 602 (2001) 585, arXiv:hep-ph/0101192. 
[18] Y. Goto, et al., Asymmetry Analysis Collaboration, Phys. Rev. D 62 (2000) 034017, arXiv:hep-ph/0001046; M. Hirai, S. Kumano, N. Saito, Asymmetry Analysis Collaboration, Phys. Rev. D 69 (2004) 054021, arXiv: hep-ph/0312112;

M. Hirai, S. Kumano, N. Saito, Phys. Rev. D 74 (2006) 014015, arXiv: hep-ph/0603213;

M. Hirai, S. Kumano, Asymmetry Analysis Collaboration, Nucl. Phys. B 813 (2009) 106, arXiv:0808.0413 [hep-ph].

[19] M. Glück, E. Reya, M. Stratmann, W. Vogelsang, Phys. Rev. D 63 (2001) 094005, arXiv:hep-ph/0011215.

[20] C.R.V. Bourrely, J. Soffer, F. Buccella, Eur. Phys. J. C 41 (2005) 327, arXiv:hep-ph/0502180.

[21] E. Leader, A.V. Sidorov, D.B. Stamenov, Eur. Phys. J. C 23 (2002) 479, arXiv:hep-ph/0111267.

[22] E. Leader, A.V. Sidorov, D.B. Stamenov, Phys. Rev. D 80 (2009) 054026, arXiv:0908.2390 [hep-ph].

[23] J. Blümlein, H. Böttcher, Nucl. Phys. B 636 (2002) 225, arXiv:hep-ph/0203155.

[24] S. Atashbar Tehrani, A.N. Khorramian, JHEP 0707 (2007) 048, arXiv:0705.2647 [hep-ph].

[25] D. de Florian, R. Sassot, M. Stratmann, W. Vogelsang, Phys. Rev. Lett. 101 (2008) 072001, arXiv:0804.0422 [hep-ph];

D. de Florian, R. Sassot, M. Stratmann, W. Vogelsang, Phys. Rev. D 80 (2009) 034030, arXiv:0904.3821 [hep-ph].

[26] B. Adeva, et al., Spin Muon Collaboration, Phys. Rev. D 58 (1998) 112002.

[27] L. Del Debbio, A. Guffanti, A. Piccione, JHEP 0911 (2009) 060, arXiv:0907.2506 [hep-ph].

[28] J. Blümlein, H. Böttcher, A. Guffanti, Nucl. Phys. B 774 (2007) 182, arXiv:hep-ph/0607200; J. Blümlein, H. Böttcher, A. Guffanti, Nucl. Phys. B (Proc. Suppl.) 135 (2004) 152, arXiv:hep-ph/0407089.

[29] M. Glück, E. Reya, C. Schuck, Nucl. Phys. B 754 (2006) 178, arXiv:hep-ph/0604116; P. Jimenez-Delgado, E. Reya, Phys. Rev. D 79 (2009) 074023, arXiv:0810.4274 [hep-ph].

[30] A.D. Martin, W.J. Stirling, R.S. Thorne, G. Watt, Eur. Phys. J. C 64 (2009) 653, arXiv:0905.3531 [hep-ph].

[31] S. Alekhin, J. Blümlein, S. Klein, S. Moch, Phys. Rev. D 81 (2010) 014032, arXiv:0908.2766 [hep-ph].

[32] A.D. Watson, Z. Phys. C 12 (1982) 123; W. Vogelsang, Z. Phys. C 50 (1991) 275.

[33] M. Buza, Y. Matiounine, J. Smith, et al., Nucl. Phys. B 485 (1997) 420, arXiv:hep-ph/9608342; I. Bierenbaum, J. Blümlein, S. Klein, arXiv:0706.2738 [hep-ph], and in preparation.

[34] S.I. Alekhin, J. Blümlein, Phys. Lett. B 594 (2004) 299, arXiv:hep-ph/0404034.

[35] M. Lacombe, B. Loiseau, R. Vinh Mau, J. Cote, P. Pires, R. de Tourreil, Phys. Lett. B 101 (1981) 139; W.W. Buck, F. Gross, Phys. Rev. D 20 (1979) 2361;

M.J. Zuilhof, J.A. Tjon, Phys. Rev. C 22 (1980) 2369;

R. Machleidt, K. Holinde, C. Elster, Phys. Rep. 149 (1987) 1;

A.Y. Umnikov, L.P. Kaptari, K.Y. Kazakov, F.C. Khanna, arXiv:hep-ph/9410241.

[36] R. Mertig, W.L. van Neerven, Z. Phys. C 70 (1996) 637, arXiv:hep-ph/9506451;

W. Vogelsang, Phys. Rev. D 54 (1996) 2023, arXiv:hep-ph/9512218.

[37] See e.g. W. Furmanski, R. Petronzio, Z. Phys. C 11 (1982) 293, and references therein; G.T. Bodwin, J.W. Qiu, Phys. Rev. D 41 (1990) 2755.

[38] E.B. Zijlstra, W.L. van Neerven, Nucl. Phys. B 417 (1994) 61; E.B. Zijlstra, W.L. van Neerven, Nucl. Phys. B 426 (1994) 245, Erratum;

E.B. Zijlstra, W.L. van Neerven, Nucl. Phys. B 773 (2007) 105, Erratum.

[39] J. Blümlein, S. Kurth, Phys. Rev. D 60 (1999) 014018, arXiv:hep-ph/9810241; J.A.M. Vermaseren, Int. J. Mod. Phys. A 14 (1999) 2037, arXiv:hep-ph/9806280.

[40] J. Blümlein, Comput. Phys. Commun. 133 (2000) 76, arXiv:hep-ph/0003100; J. Blümlein, S.O. Moch, Phys. Lett. B 614 (2005) 53, arXiv:hep-ph/0503188;

J. Blümlein, Comput. Phys. Commun. 180 (2009) 2218, arXiv:0901.3106 [hep-ph];

J. Blümlein, arXiv:0901.0837 [math-ph];

J. Ablinger, J. Blümlein, C. Schneider, in preparation.

[41] M. Glück, E. Reya, A. Vogt, Z. Phys. C 48 (1990) 471.

[42] J. Blümlein, A. Vogt, Phys. Rev. D 58 (1998) 014020, arXiv:hep-ph/9712546.

[43] J. Blümlein, A. Vogt, Phys. Lett. B 370 (1996) 149, arXiv:hep-ph/9510410; J. Blümlein, A. Vogt, Phys. Lett. B 386 (1996) 350, arXiv:hep-ph/9606254.

[44] J. Blümlein, A. Vogt, Acta Phys. Polon. B 27 (1996) 1309, arXiv:hep-ph/9603450; J. Bartels, B.I. Ermolaev, M.G. Ryskin, Z. Phys. C 72 (1996) 627, arXiv:hep-ph/9603204;

Y. Kiyo, J. Kodaira, H. Tochimura, Z. Phys. C 74 (1997) 631, arXiv:hep-ph/9701365;

J. Blümlein, arXiv:hep-ph/9909449;

J. Blümlein, Lecture Notes in Physics, vol. 546, Springer, Berlin, 2000, p. 42. 
[45] M.J. Alguard, et al., E80 Collaboration, Phys. Rev. Lett. 37 (1976) 1261;

G. Baum, et al., E130 Collaboration, Phys. Rev. Lett. 51 (1983) 1135.

[46] L.W. Withlow, et al., Phys. Lett. B 250 (1990) 193.

[47] M. Arneodo, et al., NMC Collaboration, Nucl. Phys. B 483 (1997) 3.

[48] K. Abe, et al., E143 Collaboration, Phys. Lett. B 452 (1999) 194.

[49] M. Arneodo, et al., NMC Collaboration, Phys. Lett. B 364 (1995) 107.

[50] D. Adams, et al., SMC Collaboration, Phys. Lett. B 336 (1994) 125.

[51] K. Abe, et al., E154 Collaboration, Phys. Lett. B 404 (1997) 377.

[52] K. Abe, et al., E155x Collaboration, Phys. Lett. B 553 (2003) 18.

[53] S. Wandzura, F. Wilczek, Phys. Lett. B 72 (1977) 195.

[54] A. Piccione, G. Ridolfi, Nucl. Phys. B 513 (1998) 301, arXiv:hep-ph/9707478.

[55] R.G. Roberts, G.G. Ross, Phys. Lett. B 373 (1996) 235, arXiv:hep-ph/9601235;

J. Blümlein, N. Kochelev, Nucl. Phys. B 498 (1997) 285, arXiv:hep-ph/9612318;

J. Blümlein, N. Kochelev, Phys. Lett. B 381 (1996) 296, arXiv:hep-ph/9603397.

[56] J. Blümlein, D. Robaschik, Nucl. Phys. B 581 (2000) 449, arXiv:hep-ph/0002071.

[57] J. Blümlein, D. Robaschik, Phys. Rev. D 65 (2002) 096002, arXiv:hep-ph/0202077.

[58] J. Blümlein, V. Ravindran, W.L. van Neerven, Phys. Rev. D 68 (2003) 114004, arXiv:hep-ph/0304292.

[59] J. Blümlein, A. Tkabladze, Nucl. Phys. B (Proc. Suppl.) 79 (1999) 541, arXiv:hep-ph/9905524.

[60] F. James, CERN Program Library, Long Writeup D506.

[61] J. Pumplin, D.R. Stump, J. Huston, et al., JHEP 0207 (2002) 012, arXiv:hep-ph/0201195.

[62] A. Airapetian, et al., HERMES Collaboration, Phys. Rev. D 71 (2005) 012003, arXiv:hep-ex/0407032.

[63] C. Amsler, et al., Particle Data Group, Phys. Lett. B 667 (2008) 1.

[64] M. Glück, E. Reya, A. Vogt, Eur. Phys. J. C 5 (1998) 461, arXiv:hep-ph/9806404.

[65] S. Bethke, Eur. Phys. J. C 64 (2009) 689, arXiv:0908.1135 [hep-ph].

[66] F.D. Aaron, et al., H1 Collaboration, ZEUS Collaboration, JHEP 1001 (2010) 109, arXiv:0911.0884 [hep-ex].

[67] S.I. Alekhin, J. Blümlein, S.-O. Moch, DESY 10-065, in preparation.

[68] V. Radescu et al., H1 and ZEUS Collaborations, Combined H1 and ZEUS fits using low energy data, talk, DIS 2010, Florence, April 2010.

[69] R. Abbate, M. Fickinger, A. Hoang, V. Mateu, I.W. Stewart, arXiv:1004.4894 [hep-ph];

R. Abbate, M. Fickinger, A. Hoang, V. Mateu, I.W. Stewart, arXiv:1006.3080 [hp-ph].

[70] T. Gehrmann, M. Jaquier, G. Luisoni, Eur. Phys. J. C 67 (2010) 57, arXiv:0911.2422 [hep-ph].

[71] D.B. Renner, arXiv:1002.0925 [hep-lat].

[72] H.W. Lin, T. Blum, S. Ohta, S. Sasaki, T. Yamazaki, Phys. Rev. D 78 (2008) 014505, arXiv:0802.0863 [hep-lat].

[73] Y. Aoki, et al., Phys. Rev. D 72 (2005) 114505, arXiv:hep-lat/0411006;

J. Zanotti, private communication for QCDSF;

T. Yamazaki, et al., Phys. Rev. D 79 (2009) 114505, arXiv:0904.2039 [hep-lat];

C. Gattringer, C. Hagen, C.B. Lang, M. Limmer, D. Mohler, A. Schäfer, Phys. Rev. D 79 (2009) 054501, arXiv:0812.1681 [hep-lat];

D. Mohler, private communication for BGR;

J. Negele, private communication for LHPC;

T. Korzec, private communication for ETMC.

[74] Ph. Hägler, et al., LHPC Collaborations, Phys. Rev. D 77 (2008) 094502, arXiv:0705.4295 [hep-lat];

J.D. Bratt, et al., LHPC Collaboration, arXiv:1001.3620 [hep-lat].

[75] G. Schierholz, private communication.

[76] M. Göckeler, et al., QCDSF Collaboration, Phys. Rev. D 53 (1996) 2317;

M. Göckeler, et al., Phys. Lett. B 414 (1997) 340, arXiv:hep-ph/9711245;

M. Göckeler, et al., Phys. Rev. D 63 (2001) 074506;

S. Capitani, et al., Nucl. Phys. B (Proc. Suppl.) 79 (1999) 548;

S. Güsken, et al., SESAM Collaboration, arXiv:hep-lat/9901009;

D. Dolgov, et al., LHPC Collaboration, TXL Collaboration, Phys. Rev. D 66 (2002) 034506, arXiv:hep-lat/0201021.

[77] M. Virchaux, A. Milsztajn, Phys. Lett. B 274 (1992) 221.

[78] S.I. Alekhin, Phys. Rev. D 68 (2003) 014002, arXiv:hep-ph/0211096.

[79] J. Blümlein, H. Böttcher, Phys. Lett. B 662 (2008) 336, arXiv:0802.0408 [hep-ph].

[80] S.I. Alekhin, S. Kulagin, R. Petti, The low-Q deep-inelastic scattering data in the global fit of PDFs, in: Proc. 15th International Workshop on Deep-Inelastic Scattering and Related Subjects (DIS 2007), vol. 1 Munich, Germany, 2007, p. 313. 